\title{
Isogeometric collocation methods for Cosserat rods and rod structures
}

\author{
Oliver Weeger*, Sai-Kit Yeung, Martin L. Dunn \\ Singapore University of Technology and Design, \\ SUTD Digital Manufacturing and Design Centre, \\ 8 Somapah Road, Singapore 487372, Singapore
}

\begin{abstract}
We present a novel method for the mechanical simulation of slender, elastic, spatial rods and rod structures subject to large deformation and rotation. We develop an isogeometric collocation method for the geometrically exact, nonlinear Cosserat rod theory. The rod centerlines are represented as spatial NURBS curves and cross-section orientations are parameterized in terms of unit quaternions as 4-dimensional NURBS curves. Within the isogeometric framework, the strong forms of the equilibrium equations of forces and moments of the discretized Cosserat model are collocated, leading to an efficient method for higher-order discretizations. For rod structures consisting of multiple, connected rods we introduce a formulation with rigid, quasi- $G^{1}$-coupling. It is based on the strong enforcement of continuity of displacement and change of cross-section orientation at interfaces. We also develop a mixed isogeometric formulation, which is based on an independent discretization of internal forces and moments and alleviates shear locking for thin rods. The novel rod simulation methods are verified by numerical convergence studies. Further computational examples include realistic applications with large deformations and rotations, as well as a large-scale rod structure with several hundreds of coupled rods and complex buckling behavior.
\end{abstract}

Keywords: Isogeometric analysis, Collocation method, NURBS basis functions, Cosserat rod model, Rod structures, Locking-free methods

\section{Introduction}

Isogeometric analysis is a novel concept in computational simulation, which aims at bridging the gap between the two domains of computer-aided design (CAD) and computational analysis. The main idea behind isogeometric analysis (IGA) is to employ spline-based geometry descriptions, which are typically used in CAD, also within numerical discretization methods. IGA was first introduced by Hughes et al. in 2005 [1] and has since attracted increasing interest in both communities [2].

Applications of isogeometric methods in computational mechanics include, to name a few, incompressible elasticity and plasticity [3,6], mechanical contact formulations 7,8 , nonlinear vibration analysis 9 , shape optimization [10, 11, and fluid-structure interaction [12. In particular, it has been shown analytically [13 16] and in many of these applications that isogeometric Galerkin methods provide a higher per degree of freedom (DOF) accuracy than conventional finite element methods (FEM). However, especially for 3dimensional simulations, the application of isogeometric methods still requires special modeling approaches, such as T-Splines [17, or 3D segmentation methods [18. Thus isogeometric analysis is most beneficial, when it is applied to slender structures such as beams and rods [19 23, as well as plates and shells [24 26], which allow a more straight-forward integration of design and analysis.

\footnotetext{
${ }^{*}$ Corresponding author

Email addresses: oliver_weeger@sutd.edu.sg (Oliver Weeger), saikit@sutd.edu.sg (Sai-Kit Yeung), martin_dunn@sutd.edu.sg (Martin L. Dunn)
} 
More recently, isogeometric collocation methods have been proposed as an alternative to conventional Galerkin methods [27, 28. In contrast to FEM, collocation methods are based on the strong form of the partial differential equation to be discretized, and thus require a higher continuity of the employed discrete function spaces - which can be achieved by higher-order spline spaces in isogeometric methods. It has already been shown that collocation methods are computationally more efficient than Galerkin methods 29. Isogeometric collocation schemes have been successfully developed for a wide range of applications, such as elastostatics and dynamics [30, contact problems [31, various beam and rod formulations [32 35], as well as plates and shells [35, 36].

Due to the advantages of isogeometric collocation methods for slender structures, we develop an isogeometric collocation method for the Cosserat rod model in this paper. The geometrically exact Cosserat rod model can be used to simulate mechanical large deformation and rotation behavior of 3-dimensional, slender, elastic rods [37 39]. Applications therefore include curved structures in architecture, design and engineering, such as cables, fibers, hair, and lattices. While previous numerical methods proposed from the fields of computational mechanics [40,44], computer graphics [45, 47, or multibody dynamics [48, 49, are typically based on finite element or piecewise linear discretizations of the Cosserat rod, we employ for the first time a higher-order NURBS parameterization in combination with the collocation approach.

The outline of this paper is as follows. In Section 2 we introduce the Cosserat model for the mechanics of 3-dimensional, slender, elastic rods. We present the strong form of the governing equations, i.e. the equilibria of linear and angular momentum, in terms of the centerline and rotation quaternions, which define the configuration of the Cosserat rod. Then we describe the isogeometric parameterization of the rods in Section 3. First we give a brief introduction to B-Splines and NURBS curves, and then we outline the parameterization of centerline curve and rotation quaternions using NURBS. In Section 4 we apply the principle of isogeometric collocation to the strong form of the Cosserat rod equilibrium equations. We outline the numerical discretization and its linearization in detail. For the simulation of rod structures, we introduce a rod coupling method in Section 5 , which enforces continuous centerline deformations and changes of rotation at the interfaces. To address the phenomenon of shear locking of thin rods, we furthermore develop a mixed isogeometric collocation scheme in Section 6, which is based on an independent NURBS discretization of internal forces and moments. In Section 7 we validate the presented methods using numerical benchmark examples and show the application to complex problems with large deformations and rotations, as well as rod structures. Finally, a summary and conclusions are given in 8 .

\section{Cosserat rod model}

A rod is a long and thin deformable body, with its length being significantly longer than the cross-section diameter. The Cosserat rod theory is an approach to the modeling of slender elastic rods, which assumes that the cross-sections themselves remain undeformed under loading. It can be interpreted as a nonlinear extension of 3-dimensional Timoshenko beam theory [38, 49]. In the Euler-Bernoulli beam and Kirchhoff rod theory cross-sections have to remain normal to the centerline deformation. Thus shear deformation is neglected and the theory is only valid for thin beams. On the contrary, the Cosserat rod and Timoshenko beam theories also assume that the cross-sections remain straight, but not necessarily normal to the tangent of the centerline curve, thus accounting also for shear deformation. In the following we outline Cosserat rod theory in more detail based on 37, 39.

\subsection{Configuration of the rod}

The Cosserat theory is based on the description of the configuration of the rod as a framed curve. The rod is represented by the line of its mass centroids, or simply its centerline, which is a spatial curve:

$$
\mathbf{r}:[0, L] \rightarrow \mathbb{R}^{3} .
$$

This curve is assumed to be arc-length parameterized in the initial configuration, which means that

$$
\|\mathbf{t}(s)\|=1 \forall s \in[0, L],
$$




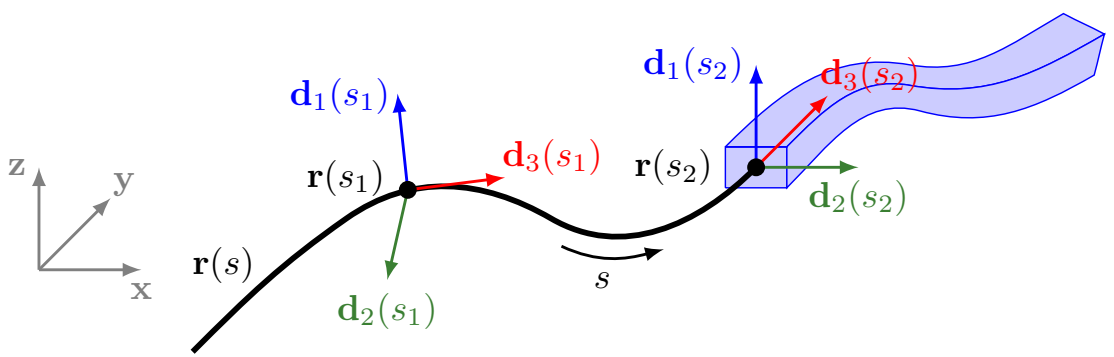

Figure 1: Configuration of a Cosserat rod, which is represented by its centerline $\mathbf{r}(s)$ and orthonormal frames $\mathbf{R}(s)=$ $\left(\mathbf{d}_{1}(s), \mathbf{d}_{2}(s), \mathbf{d}_{3}(s)\right)$, which define the cross-section orientations

where

$$
\mathbf{t}(s)=\mathbf{r}^{\prime}(s)=\frac{d \mathbf{r}}{d s} .
$$

is its tangent vector at parameter value $s$ and

$$
L=\int_{0}^{L}\left\|\mathbf{r}^{\prime}(s)\right\| d s
$$

is the length of the curve.

Furthermore, a frame, i.e. a local orthonormal basis field, is needed to describe the evolution of the orientation of the cross-sections along the rod:

$$
\mathbf{R}:[0, L] \rightarrow S O(3) .
$$

$S O(3)$ is the group of rotations in the 3 -dimensional Euclidean space $\mathbb{R}^{3}$ and thus these local frames can be associated with $3 \mathrm{D}$ rotation matrices:

$$
\mathbf{R}(s)=\left(\mathbf{d}_{1}(s), \mathbf{d}_{2}(s), \mathbf{d}_{3}(s)\right) \in \mathbb{R}^{3 \times 3}: \quad \mathbf{R}^{\top} \mathbf{R}=\mathbf{I}, \operatorname{det} \mathbf{R}=1 \forall s \in[0, L] .
$$

The representation of a rod using its centerline curve and frames completely determines its configuration and is illustrated in Fig. 1 .

\subsection{Parameterization and initialization of rotations}

Since $3 \times 3$-rotation matrices have 9 components, but only 3 independent degrees of freedom, a number of possibilities exists for their parameterization. Popular choices are Euler angles, rotation vectors or axis and angle representation. Here we adopt the parameterization using unit quaternions, which is commonly used in Cosserat rod literature [4, 49.

Unit quaternions are defined as normalized quadruples of real numbers, i.e.

$$
\mathbf{q}=\left(\begin{array}{c}
q_{1} \\
q_{2} \\
q_{3} \\
q_{4}
\end{array}\right) \in \mathbb{R}^{4}:\|\mathbf{q}\|=1
$$

and parameterize rotation matrices as:

$$
\mathbf{q} \rightarrow \mathbf{R}(\mathbf{q}) \in S O(3): \mathbf{R}(\mathbf{q})=\left(\begin{array}{ccc}
q_{1}^{2}-q_{2}^{2}-q_{3}^{2}+q_{4}^{2} & 2\left(q_{1} q_{2}-q_{3} q_{4}\right) & 2\left(q_{1} q_{3}+q_{2} q_{4}\right) \\
2\left(q_{1} q_{2}+q_{3} q_{4}\right) & -q_{1}^{2}+q_{2}^{2}-q_{3}^{2}+q_{4}^{2} & 2\left(q_{2} q_{3}-q_{1} q_{4}\right) \\
2\left(q_{1} q_{3}-q_{2} q_{4}\right) & 2\left(q_{2} q_{3}+q_{1} q_{4}\right) & -q_{1}^{2}-q_{2}^{2}+q_{3}^{2}+q_{4}^{2}
\end{array}\right) .
$$

Since in (6) the frames given by rotation matrices have to be parameterized in terms of the arc-length parameter $s$, the quaternions have to be represented in that fashion:

$$
\mathbf{q}:[0, L] \rightarrow S O(3) \leadsto \mathbf{R}(s)=\mathbf{R}(\mathbf{q}(s)) .
$$


While in the Kirchhoff rod model $\mathbf{d}_{3}$ is and remains aligned with the tangent vector $\mathbf{t}$ of the curve, the initial orientation of the cross-sections given by $\mathbf{R}(s)$ resp. $\mathbf{q}(s)$ is basically arbitrary in the Cosserat model. However, it has to be fixed in order to define the initial configuration of the rod and given solely a centerline curve $\mathbf{r}$, a natural choice for the determination of the initial frame of the rod is the Darboux frame, which forms an intrinsic orthonormal basis of cross-sections:

$$
\mathbf{d}_{3}=\mathbf{t}=\mathbf{r}^{\prime}, \quad \mathbf{d}_{1}=\mathbf{n}=\frac{\mathbf{t}^{\prime}}{\left\|\mathbf{t}^{\prime}\right\|}=\frac{\mathbf{r}^{\prime \prime}}{\left\|\mathbf{r}^{\prime \prime}\right\|}, \quad \mathbf{d}_{2}=\mathbf{b}=\mathbf{d}_{3} \times \mathbf{d}_{1} .
$$

Another option, which gives in many cases a more desirable initialization of the orientations, is the torsion free Bishop frame [50. It is defined by the relations:

$$
\mathbf{d}_{3}=\mathbf{t}=\mathbf{r}^{\prime}, \quad \kappa_{3}=\mathbf{d}_{1}^{\prime \top} \mathbf{d}_{2}=-\mathbf{d}_{2}^{\prime \top} \mathbf{d}_{1}=0
$$

It can be determined for a fixed starting point rotation $\mathbf{R}(0)$ by enforcing the following conditions for $s \in(0, L]:$

$$
\mathbf{d}_{1}^{\top} \mathbf{d}_{3}=0, \quad \mathbf{d}_{2}^{\top} \mathbf{d}_{3}=0, \quad \mathbf{d}_{1}^{\prime \top} \mathbf{d}_{2}=0 .
$$

If the cross-sections are twisted around the tangent of the curve, i.e. the principal axes of inertia of the cross-sections are not aligned with $\mathbf{d}_{1}$ and $\mathbf{d}_{2}$ as determined by the Darboux or Bishop frame, the frame can still be represented by a rotation of the Darboux or Bishop frame around the tangent $\mathbf{d}_{3}$ by an angle $\alpha \in[0,2 \pi)$.

\subsection{Kinematics and constitutive equations}

Based on the two kinds of kinematic unknowns that are needed to define the configuration of the rods, i.e. the centerline curve $\mathbf{r}:[0, L] \rightarrow \mathbb{R}^{3}$ and the quaternion field $\mathbf{q}:[0, L] \rightarrow S O(3)$ describing the orthonormal frames, the strains in the spatial or current configuration can be formulated and evaluated at $s \in[0, L]$. The translational strains are defined by the vector

$$
\varepsilon=\mathbf{R}^{\top} \mathbf{r}^{\prime}-\mathbf{e}_{3},
$$

where $\mathbf{e}_{3}$ denotes the Cartesian basis vector $(0,0,1)^{\top}$. The components $\varepsilon_{1}$ and $\varepsilon_{2}$ refer to the shear strains, while $\varepsilon_{3}$ is the axial or extensional strain. Furthermore, the rotational strains are given in terms of the curvature vector of the rod:

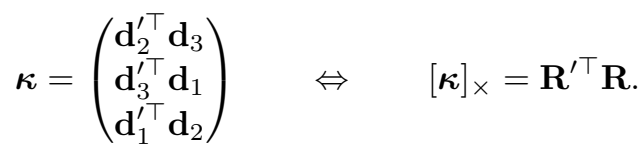

The latter expression corresponds to Frenet-Serret formulas $\mathbf{R}^{\prime \top}=[\boldsymbol{\kappa}]_{\times} \mathbf{R}^{\top}$, where $[\cdot]_{\times}$represents the skewsymmetric cross-product matrix for a 3 -dimensional vector, i.e. for $\mathbf{u}, \mathbf{v} \in \mathbb{R}^{3}$ :

$$
\mathbf{u} \times \mathbf{v}=[\mathbf{u}]_{\times} \mathbf{v} \text { with }[\mathbf{u}]_{\times}=\left(\begin{array}{ccc}
0 & u_{3} & -u_{2} \\
-u_{3} & 0 & u_{1} \\
u_{2} & -u_{1} & 0
\end{array}\right) .
$$

The components $\kappa_{1}$ and $\kappa_{2}$ are bending curvatures or flexural strains, while $\kappa_{3}$ corresponds to the torsional curvature or strain.

Based on these two nonlinear strain vectors, which both refer to the rotated local coordinate frame at $s$, the stresses in the current configuration are derived by linear elastic constitutive laws:

$$
\begin{array}{ll}
\text { translational stresses: } & \boldsymbol{\sigma}=\mathbf{C}\left(\boldsymbol{\varepsilon}-\boldsymbol{\varepsilon}_{0}\right), \\
\text { rotational stresses: } & \chi=\mathbf{D}\left(\boldsymbol{\kappa}-\boldsymbol{\kappa}_{0}\right) .
\end{array}
$$


The terms $\varepsilon_{0}$ and $\boldsymbol{\kappa}_{0}$ represent strains in the initial configuration $\left(\mathbf{r}_{0}, \mathbf{R}_{0}\right)$, which might for instance result from pre-stretching or misalignment of $\mathbf{t}$ and $\mathbf{d}_{3}$ in the case of $\boldsymbol{\varepsilon}_{0} \neq \mathbf{0}$ and curvature of initially curved rods in the case of $\boldsymbol{\kappa}_{0} \neq \mathbf{0}$. Furthermore, in 16 the intrinsic material matrices are used:

$$
\mathbf{C}=\left(\begin{array}{ccc}
k_{1} G A & 0 & 0 \\
0 & k_{2} G A & 0 \\
0 & 0 & E A
\end{array}\right), \quad \mathbf{D}=\left(\begin{array}{ccc}
E I_{1} & 0 & 0 \\
0 & E I_{2} & 0 \\
0 & 0 & G J
\end{array}\right)
$$

These are given solely in terms of cross-section parameters, i.e. material constants, namely elastic modulus $E$ and shear modulus $G=E /(2+2 \nu)$ with Poisson's ratio $\nu$, and geometrical or shape constants, namely cross section area $A$, second moments of area $I_{1}$ and $I_{2}$, torsion constant $J$ (typically $J=I_{1}+I_{2}$ ), and shear correction factors $k_{1}$ and $k_{2}$ (typically $k_{1,2}=5 / 6$ ).

\subsection{Equilibrium equations}

Before the balance equations of linear and angular momentum can be formulated, the stress vectors introduced above in (16) have to be rotated into the global Euclidian coordinate frame. This transformation from the spatial back to the material configuration results in the expressions for internal force and moment vectors:

$$
\begin{array}{rlrl} 
& \text { internal forces: } & \mathbf{n} & =\mathbf{R} \boldsymbol{\sigma}=\mathbf{R C}\left(\varepsilon-\varepsilon_{0}\right), \\
\text { internal moments: } & \mathbf{m} & =\mathbf{R} \boldsymbol{\chi}=\mathbf{R D}\left(\boldsymbol{\kappa}-\boldsymbol{\kappa}_{0}\right) .
\end{array}
$$

Then the equilibrium equations of linear and angular momentum in material/initial configuration can be formulated as:

$$
\begin{aligned}
\mathbf{n}^{\prime}+\hat{\mathbf{n}}=\mathbf{0} & \forall s \in(0, L), \\
\mathbf{m}^{\prime}+\mathbf{r}^{\prime} \times \mathbf{n}+\hat{\mathbf{m}}=\mathbf{0} & \forall s \in(0, L) .
\end{aligned}
$$

Here $\hat{\mathbf{n}}$ and $\hat{\mathbf{m}}$ are external distributed forces and moments applied on the rod. Furthermore, at the two ends of the rod, $s=0$ and $s=L$, boundary conditions either of Dirichlet- or Neumann-type have to be applied. Dirichlet or displacement/rotation boundary conditions are of the form

$$
\mathbf{r}=\overline{\mathbf{r}} \quad \wedge \quad \mathbf{q}=\overline{\mathbf{q}}, \quad s=0, L,
$$

and Neumann or force/moment boundary conditions read

$$
\mathbf{n}=\overline{\mathbf{n}} \quad \wedge \quad \mathbf{m}=\overline{\mathbf{m}}, \quad s=0, L,
$$

where $\overline{\mathbf{r}}, \overline{\mathbf{q}}, \overline{\mathbf{n}}$, and $\overline{\mathbf{m}}$ refer to the prescribed endpoint displacement, rotation, force, or moment.

Since we are using quaternions for the parameterization of rotations, additionally a unit length constraint for quaternions must hold to complete the equilibrium equations:

$$
\mathbf{q}^{\top} \mathbf{q}-1=0 \quad \forall s \in[0, L] .
$$

Altogether, the equilibrium equations of linear and angular momentum (19), together with boundary conditions 20 or $(21)$ and the unit-length constraint for quaternions 22, form a nonlinear system of 7 equations in the 7 kinematic unknowns $\mathbf{r}$ and $\mathbf{q}$, which must hold for all $s \in[0, L]$.

For a better understanding and implementation purposes, we formulate the expressions in above-mentioned equilibrium equations in more detail:

$$
\begin{aligned}
\mathbf{n}^{\prime} & =\mathbf{R}^{\prime} \boldsymbol{\sigma}+\mathbf{R} \boldsymbol{\sigma}^{\prime} \\
& =\mathbf{R}^{\prime} \mathbf{C}\left(\varepsilon-\varepsilon_{0}\right)+\mathbf{R C}\left(\varepsilon^{\prime}-\varepsilon_{0}^{\prime}\right) \\
& =\mathbf{R}^{\prime} \mathbf{C}\left(\mathbf{R}^{\top} \mathbf{r}^{\prime}-\mathbf{e}_{3}-\varepsilon_{0}\right)+\mathbf{R C}\left(\mathbf{R}^{\prime \top} \mathbf{r}^{\prime}+\mathbf{R}^{\top} \mathbf{r}^{\prime \prime}-\varepsilon_{0}^{\prime}\right) \\
\mathbf{m}^{\prime}+\mathbf{r}^{\prime} \times \mathbf{n} & =\mathbf{R}^{\prime} \chi+\mathbf{R} \chi^{\prime}+\mathbf{r}^{\prime} \times(\mathbf{R} \boldsymbol{\sigma}) \\
& =\mathbf{R}^{\prime} \mathbf{D}\left(\boldsymbol{\kappa}-\boldsymbol{\kappa}_{0}\right)+\mathbf{R D}\left(\boldsymbol{\kappa}^{\prime}-\boldsymbol{\kappa}_{0}^{\prime}\right)+\mathbf{r}^{\prime} \times\left(\mathbf{R C}\left(\varepsilon-\varepsilon_{0}\right)\right) \\
& =\mathbf{R}^{\prime} \mathbf{D}\left(\boldsymbol{\kappa}-\boldsymbol{\kappa}_{0}\right)+\mathbf{R D}\left(\boldsymbol{\kappa}^{\prime}-\boldsymbol{\kappa}_{0}^{\prime}\right)+\mathbf{r}^{\prime} \times\left(\mathbf{R C}\left(\mathbf{R}^{\top} \mathbf{r}^{\prime}-\mathbf{e}_{3}-\varepsilon_{0}\right)\right)
\end{aligned}
$$




\section{Isogeometric parameterization of the rod model}

In this Section we apply the concept of isogeometric analysis to the Cosserat rod problem. The main idea behind isogeometric methods, as introduced in 1, is that both the geometry and numerical solution are discretized in terms of spline basis functions.

\subsection{NURBS basis functions and curves}

B-Splines and Non-Uniform Rational B-Splines (NURBS) are widely used for describing geometries in computer-aided design and also for numerical discretizations in isogeometric analysis [1, 2, Here we briefly review their basic definitions and properties, which can be found in much more detail in [51].

B-Splines are piecewise polynomial functions, which are defined using a knot vector $\Xi=\left\{\xi_{1}, \ldots, \xi_{m}\right\}$, which is a non-decreasing sequence of knots $\xi_{i} \in \mathbb{R}(i=1, \ldots, m), \xi_{i} \leq \xi_{i+1}(i=1, \ldots, m-1)$ on the parameter domain $\Omega_{0}=\left[\xi_{1}, \xi_{m}\right] \subset \mathbb{R}$. For two distinct knots $\xi_{i} \neq \xi_{i+1}$, the half-open interval $\left[\xi_{i}, \xi_{i+1}\right)$ is called the $i$-th knot span or element. The total number of nonzero knot spans or elements in $\Xi$ is denoted by $\ell$. Here we only use open knot vectors, where the first and last knot have multiplicity $p+1$, with inner knots of multiplicity $1 \leq k \leq p$.

The B-Spline basis functions $B_{i}^{p}(\xi): \Omega_{0} \rightarrow[0,1]$ of degree $p$ and order $p+1$ on the knot vector $\Xi$ are defined for $i=1, \ldots, n(n=m-p-1)$ by the Cox-de Boor recursion:

$$
B_{i}^{0}(\xi)=\left\{\begin{array}{ll}
1 & \xi_{i} \leq \xi<\xi_{i+1} \\
0 & \text { else }
\end{array}, \quad B_{i}^{p}(\xi)=\frac{\xi-\xi_{i}}{\xi_{i+p}-\xi_{i}} B_{i}^{p-1}(\xi)+\frac{\xi_{i+p+1}-\xi}{\xi_{i+p+1}-\xi_{i+1}} B_{i+1}^{p-1}(\xi)\right.
$$

Here the quotient $0 / 0$ is defined to be 0 .

Non-Uniform Rational B-Spline (NURBS) basis functions $N_{i}^{p}$ are piecewise rational functions of degree $p$, which are defined using B-Spline basis functions $B_{i}^{p}$ on a knot vector $\Xi$ and additional weights $w_{i}>0(i=$ $1, \ldots, n)$ :

$$
N_{i}^{p}(\xi)=\frac{B_{i}^{p}(\xi) w_{i}}{\sum_{j=1}^{n} B_{j}^{p}(\xi) w_{j}}
$$

For equal weights, i.e. $w_{i}=$ const. $\forall i=1, \ldots, n$, NURBS reduce to B-Spline basis functions and thus in the following we are mainly using the term NURBS. Furthermore, we drop the superscript $p$ and denote NURBS basis functions only as $N_{i}$.

There are many useful properties of B-Spline and NURBS basis functions, such as compact support, i.e. $\operatorname{supp}\left(N_{i}\right)=\left[\xi_{i}, \xi_{i+p+1}\right)$, non-negativity, i.e. $N_{i}(\xi) \geq 0 \forall \xi \in\left[\xi_{1}, \xi_{m}\right]$, partition of unity for open knot vectors, i.e. $\sum_{i=1}^{n} N_{i}(\xi) \equiv 1 \forall \xi \in\left[\xi_{1}, \xi_{m}\right]$, and smoothness, i.e. they are $p$-times continuously differentiable ( $C^{p}$-continuous) inside a knot span and at inner knots of multiplicity $k$ only $C^{p-k}$. Furthermore, we define a spline function space as $\mathcal{S}_{\Xi}=\operatorname{span}\left\{N_{i}, i=1, \ldots, n\right\}$.

Univariate B-Splines and NURBS can then be used to define geometric entities such as curves. A spline curve $\mathbf{c}: \Omega_{0} \rightarrow \mathbb{R}^{d}$ is defined by a spline space $\mathcal{S}_{\Xi}$ and control points $\mathbf{c}_{i} \in \mathbb{R}^{d}(i=1, \ldots, n)$ :

$$
\mathbf{c}(\xi)=\sum_{i=1}^{n} N_{i}(\xi) \mathbf{c}_{i}
$$

Some important properties of spline curves on open knot vectors are the convex hull property, i.e. the curve is completely contained in its control polygon, interpolation of start and end points, i.e. $\mathbf{c}\left(\xi_{1}\right)=\mathbf{c}_{1}, \mathbf{c}\left(\xi_{m}\right)=\mathbf{c}_{n}$, local control, i.e. a control point $\mathbf{c}_{i}$ only influences the curve locally in the knot interval $\left[\xi_{i}, \xi_{i+p+1}\right)$, and that affine transformations of the curve can be performed directly on its control points. Furthermore, the continuity properties of the curve correspond to the ones of NURBS functions. 


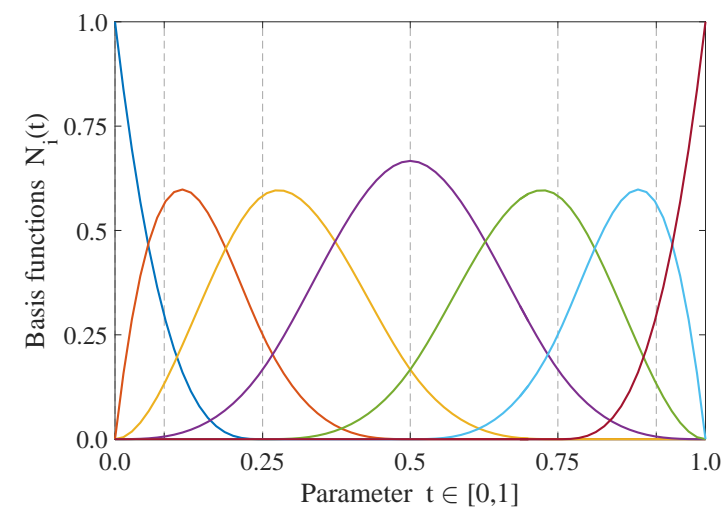

(a) B-Spline basis functions

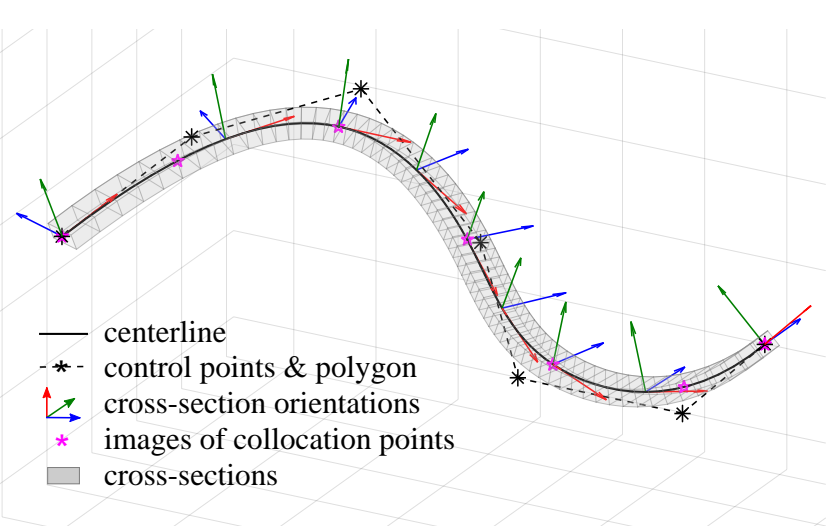

(b) Isogeometric parameterization of rod with B-Spline curve

Figure 2: Isogeometric rod parameterization; cubic B-Spline basis functions as shown in (a) with $p=3, m=11, n=7, \Xi=$ $\left\{0,0,0,0, \frac{1}{4}, \frac{1}{2}, \frac{3}{4}, 1,1,1,1\right\}$ are used for the isogeometric parameterization of the Cosserat rod in (b) with centerline and rotation quaternions as B-Spline curves

\subsection{Parameterization of the rods}

Having introduced NURBS basis functions and NURBS curves, we now derive the isogeometric parameterization of the Cosserat rod model. This means that both kinematic unknowns, the centerline curve of the rod and the quaternion field defining the cross-section orientations, are parameterized as NURBS curves.

Using $n$ basis functions $\left\{N_{i}\right\}_{i=1, \ldots, n}$ of degree $p$, defined on a knot vector $\Xi=\left\{\xi_{1}, \ldots, \xi_{m}\right\}, \xi_{i} \in$ $[0,1], m=n+p+1$, and control points $\left\{\mathbf{r}_{0, i}\right\}_{i=1, \ldots, n}, \mathbf{r}_{0, i} \in \mathbb{R}^{3}$, we write the initial, undeformed centerline curve $\mathbf{r}_{0}$ of a rod as a 3 -dimensional spline curve:

$$
\mathbf{r}_{0}:[0,1] \rightarrow \mathbb{R}^{3}, \quad \mathbf{r}_{0}(t)=\sum_{i=1}^{n} N_{i}(t) \mathbf{r}_{0, i}
$$

Furthermore, the quaternion field $\mathbf{q}_{0}$ for the representation of the initial cross-section orientations $\mathbf{R}_{0}$ are parameterized as a 4 -dimensional spline curves with control points $\left\{\mathbf{q}_{0, i}\right\}_{i=1, \ldots, n}, \mathbf{q}_{0, i} \in \mathbb{R}^{4}$ :

$$
\mathbf{q}_{0}:[0,1] \rightarrow \mathbb{R}^{4}, \quad \mathbf{q}_{0}(t)=\sum_{i=1}^{n} N_{i}(t) \mathbf{q}_{0, i}
$$

Without loss of generality we are assuming here that $\Omega_{0}=[0,1]$. Furthermore, the same spline space $\mathcal{S}_{\Xi}$ is used for both centerline and quaternions, but in general it would be possible to parameterize both using two different spline spaces, see also 33 .

As an example, in Fig. 2 a rod is parameterized using cubic B-Spline basis functions $(p=3)$ with $n=7$ control points and $\ell=4$ elements in the knot vector $\Xi=\left\{0,0,0,0, \frac{1}{4}, \frac{1}{2}, \frac{3}{4}, 1,1,1,1\right\}$. Figure $2 \mathrm{a}$ shows the basis functions and Fig. $2 \mathrm{~b}$ the rod, which is given in terms of its centerline curve and rotation quaternion curve, which defines the orientation of the rectangular cross-section.

Remark (spline parameterization of quaternions):

In equations (7)-(9) unit quaternions are used to define the rotations $\mathbf{R}(\mathbf{q})$ as a map $[0, L] \rightarrow S O(3)$, but above in (28) we have parameterized the quaternions for the initial rotations $\mathbf{R}_{0}$ as a spline curve $[0, L] \rightarrow \mathbb{R}^{4}$. Thus we need the additional condition $\|\mathbf{q}(s)\|=1$ from $(22$, which ensures that the 4dimensional vectors are actually unit quaternions and thus $\mathbf{R}(\mathbf{q}) \in S O(3)$. In the setting of the isogeometric collocation method introduced in Section 4.1, we enforce this condition numerically only at the collocation points $\tau_{i}$, see $(34)_{3}$ and 35$]_{3}$. Alternatively, a consistent parameterization could be achieved using an extension of B-Splines on the quaternion vector space, so-called quaternion splines [52. However, for 
arbitrary degrees their implementation and derivation is very complex and computationally inefficient. The parameterization presented in this work can be interpreted as a simplification of quaternion splines, which is valid for small incremental changes of rotation, i.e. when the number of control points used for the parameterization of $\mathbf{q}_{0}$ is sufficiently large. As the later numerical studies will show, this assumption is justified in most applications and leads to the expected convergence behavior of the method. The authors think that a formulation based on quaternion splines could probably add more robustness to the method, e.g. enable a faster convergence and larger steps of Newton's method for very large displacements and changes of rotation.

\subsection{Arc-length parameterization}

For the introduction of the Cosserat rod theory in Section 2 we have assumed that the initial centerline curve of the rod is arc-length parameterized. However, above we have defined the centerline as a spline curve $\mathbf{r}_{0}(t):[0,1] \rightarrow \mathbb{R}^{3}$, which is general not an arc-length parameterization.

Thus we need to describe how to compute the derivatives of a vector field defined as $t \rightarrow \mathbf{y}(t):[0,1] \rightarrow \mathbb{R}^{d}$ (this could be for instance $\mathbf{y} \equiv \mathbf{r}$ with $d=3$ or $\mathbf{y} \equiv \mathbf{q}$ with $d=4$ ) with respect to the arc-length parameter $s$ of the initial curve $\mathbf{r}_{0}(t)$. Since the current arc-length is defined as

$$
s(t)=\int_{0}^{t}\left\|\dot{\mathbf{r}}_{0}(\tau)\right\| d \tau \quad \leadsto \quad \frac{d s}{d t}(t)=\left\|\dot{\mathbf{r}}_{0}(t)\right\|=: J(t),
$$

it follows for $\mathbf{y}^{\prime}$ :

$$
\mathbf{y}^{\prime}=\frac{d \mathbf{y}}{d s}=\frac{d \mathbf{y}}{d t} \frac{d t}{d s}=\dot{\mathbf{y}}\left(\frac{d s}{d t}\right)^{-1}=\dot{\mathbf{y}} \frac{1}{\left\|\dot{\mathbf{r}}_{0}(t)\right\|}=\frac{1}{J} \dot{\mathbf{y}}
$$

Here and in the following the conventions $\dot{\mathbf{y}}=\frac{d \mathbf{y}}{d t}$ for the parametric and $\mathbf{y}^{\prime}=\frac{d \mathbf{y}}{d s}$ for the arc-length derivative are used. Since the arc-length derivative has to be defined for all $t \in[0,1]$, it is required that $J>0 \forall t \in[0,1]$, i.e. the parametric derivative of the initial centerline curve must not vanish. Furthermore, for second derivatives w.r.t. $s$ it follows:

$$
\begin{aligned}
\mathbf{y}^{\prime \prime}(t) & =\frac{d^{2} \mathbf{y}}{d s^{2}}(t)=\frac{d}{d s}\left[\frac{1}{\left\|\dot{\mathbf{r}}_{0}(t)\right\|} \dot{\mathbf{y}}(t)\right]=\frac{d}{d t}\left[\frac{1}{J(t)} \dot{\mathbf{y}}(t)\right]\left(\frac{d s}{d t}\right)^{-1} \\
& =\left(\frac{1}{J} \ddot{\mathbf{y}}-\frac{\dot{\mathbf{r}}_{0}^{T} \ddot{\mathbf{r}}_{0}}{J^{3}} \dot{\mathbf{y}}\right) \frac{1}{\left\|\dot{\mathbf{r}}_{0}(t)\right\|}=\frac{1}{J^{2}} \ddot{\mathbf{y}}-\frac{\dot{\mathbf{r}}_{0}^{T} \ddot{\mathbf{r}}_{0}}{J^{4}} \dot{\mathbf{y}}
\end{aligned}
$$

For instance, it follows for the tangent and normal vectors of the current centerline curve:

$$
\mathbf{t}(t)=\mathbf{r}^{\prime}(t)=\frac{1}{\left\|\dot{\mathbf{r}}_{0}(t)\right\|} \dot{\mathbf{r}}(t)=\frac{1}{J} \dot{\mathbf{r}}, \quad \mathbf{n}(t)=\mathbf{t}^{\prime}(t)=\mathbf{r}^{\prime \prime}(t)=\frac{1}{J^{2}} \ddot{\mathbf{r}}-\frac{\dot{\mathbf{r}}_{0}^{T} \ddot{\mathbf{r}}_{0}}{J^{4}} \dot{\mathbf{r}}
$$

\subsection{Interpolation of initial rotations}

As we have already outlined in Sect. 2.2, in the Cosserat theory the initial orientation of cross-sections given by rotation matrices $\mathbf{R}_{0}$ resp. the quaternion field $\mathbf{q}_{0}$ is an input design parameter, just as the centerline curve $\mathbf{r}_{0}$.

However, in practical applications the quaternion field $\mathbf{q}(s)$ may not be given directly as a spline curve with control points $\left\{\mathbf{q}_{0, i}\right\}_{i=1, \ldots, n}$. Instead, we assume that a rotation matrix field $\mathbf{R}_{0}(s)$ is given, either directly or it has to be determined from the centerline curve $\mathbf{r}_{0}(s)$, for instance as its Darboux frame 10 or Bishop frame (11). In this case the unit quaternion field $\mathbf{q}_{0}(s)$ resp. its control points $\mathbf{q}_{0, i}$ have to be fitted such that $\mathbf{R}\left(\mathbf{q}_{0}(s)\right)$ approximates or interpolates the given $\mathbf{R}_{0}(s)$. In the context of an isogeometric collocation method, we chose to fit the $\mathbf{q}_{0, i}$ 's such that $\mathbf{q}_{0}(s)$ interpolates the unit quaternions computed from an input rotation matrix field $\mathbf{q}\left(\mathbf{R}_{0}(s)\right)$ at the $n$ collocation points (see Sect. 4.1). 


\section{Isogeometric collocation method}

Having described the Cosserat rod model in Section 2 and introduced its parameterizations using spline curves in Section 3, we now propose the application of the isogeometric collocation method [27, 28, for the numerical discretization of the balance equations of the Cosserat rod.

\subsection{Collocation of strong equilibrium equations}

Following the isoparametric concept, the unknown centerline position $\mathbf{r}$ and orientation quaternion $\mathbf{q}$ in the current/deformed configuration are also discretized as spline curves, just as their initial counterparts $\mathbf{r}_{0}$ and $\mathbf{q}_{0}$ in 27) and 28):

$$
\begin{aligned}
\mathbf{r}_{h}:[0,1] \rightarrow \mathbb{R}^{3}, & \mathbf{r}_{h}(t)=\sum_{i=1}^{n} N_{i}(t) \mathbf{r}_{i}, \\
\mathbf{q}_{h}:[0,1] \rightarrow \mathbb{R}^{4}, & \mathbf{q}_{h}(t)=\sum_{i=1}^{n} N_{i}(t) \mathbf{q}_{i} .
\end{aligned}
$$

Here the $N_{i}$ 's may be the either same basis functions as in 27) or refined versions thereof. Refinement refers to so-called $p$ - $/ h$ - $/ k$-refinement, which means increasing the degree of spline functions, subdividing the knot vectors into more elements and the combination of both respectively [1].

To determine the unknown control points of the displaced centerline and the quaternions from (33), we apply collocation of the strong form of the equilibrium equations of the Cosserat rod, which means that the equations have to be evaluated at a set of points $\left\{\tau_{i}\right\}_{i=1, \ldots, n}$. For internal collocation points $\tau_{i}, i=2, \ldots, n-1$ this means according to 19 ) and 22 :

$$
\begin{array}{ll}
\mathbf{f}_{\mathbf{n}}\left(\tau_{i}\right)=\mathbf{n}^{\prime}\left(\tau_{i}\right)+\hat{\mathbf{n}}\left(\tau_{i}\right) & =\mathbf{0}, \\
\mathbf{f}_{\mathbf{m}}\left(\tau_{i}\right)=\mathbf{m}^{\prime}\left(\tau_{i}\right)+\mathbf{r}_{h}^{\prime}\left(\tau_{i}\right) \times \mathbf{n}\left(\tau_{i}\right)+\hat{\mathbf{m}}\left(\tau_{i}\right) & =\mathbf{0}, \\
\mathbf{f}_{\mathbf{q}}\left(\tau_{i}\right)=\mathbf{q}_{h}\left(\tau_{i}\right)^{\top} \mathbf{q}_{h}\left(\tau_{i}\right)-1 & =0 .
\end{array}
$$

At the boundaries for $\tau_{1}=0$ and $\tau_{n}=1$ either centerline position $\mathbf{r}$ and rotation quaternion $\mathbf{q}$ are a priori imposed according to $(20)$, or for Neumann conditions (21) and 22 have to be collocated as well:

$$
\begin{array}{ll}
\mathbf{f}_{\mathbf{n}}\left(\tau_{i}\right)=\mathbf{n}\left(\tau_{i}\right)-\overline{\mathbf{n}}\left(\tau_{i}\right) & =\mathbf{0}, \\
\mathbf{f}_{\mathbf{m}}\left(\tau_{i}\right)=\mathbf{m}\left(\tau_{i}\right)-\overline{\mathbf{m}}\left(\tau_{i}\right) & =\mathbf{0}, \\
\mathbf{f}_{\mathbf{q}}\left(\tau_{i}\right)=\mathbf{q}_{h}\left(\tau_{i}\right)^{\top} \mathbf{q}_{h}\left(\tau_{i}\right)-1 & =0 .
\end{array}
$$

Altogether, (34) and (35) define a set of $7 \cdot n$ nonlinear equations for the $3 \cdot n$ unknown control points of $\mathbf{r}_{h}$ and $4 \cdot n$ unknowns of $\mathbf{q}_{h}$, which can be formulated in terms of a residual vector $\mathbf{f}$ as:

$$
\mathbf{f}: \mathbb{R}^{3 \cdot n} \times \mathbb{R}^{4 \cdot n} \rightarrow \mathbb{R}^{7 \cdot n}: \quad \mathbf{f}(\overrightarrow{\mathbf{r}}, \overrightarrow{\mathbf{q}})=\left(\begin{array}{c}
\mathbf{f}_{\mathbf{n}}\left(\tau_{i}\right) \\
\mathbf{f}_{\mathbf{m}}\left(\tau_{i}\right) \\
\mathbf{f}_{\mathbf{q}}\left(\tau_{i}\right)
\end{array}\right){ }_{i=1, \ldots, n}(\overrightarrow{\mathbf{r}}, \overrightarrow{\mathbf{q}})=\mathbf{0},
$$

where the control points of $\mathbf{r}_{h}$ are combined into a large vector $\overrightarrow{\mathbf{r}}=\left(\mathbf{r}_{i}\right)_{i=1, \ldots, n} \in \mathbb{R}^{3 \cdot n}$ and likewise for $\mathbf{q}_{h}$ as $\overrightarrow{\mathbf{q}}=\left(\mathbf{q}_{i}\right)_{i=1, \ldots, n} \in \mathbb{R}^{4 \cdot n}$.

The collocation points have to be carefully chosen in order to guarantee the stability of the method [27. A common choice are the Greville abscissae of the spline knot vector, which are defined for $0 \leq k<p$ as:

$$
\tau_{i}=\frac{\xi_{i+1+k}+\ldots+\xi_{i+p}}{p-k}, i=1, \ldots, n-k .
$$

Since we have $n-2$ internal and 2 boundary collocation points, we use the $n$ Greville abscissae of order $k=0$ here. 


\subsection{Linearization}

To determine the deformed configuration of the rod for given distributed and boundary forces and moments, the solution $(\overrightarrow{\mathbf{r}}, \overrightarrow{\mathbf{q}})$ of the nonlinear system $(36)$ has to be computed. This is done by using a Newton's method, which requires the linearization of $(36)$, i.e. the derivatives of $\mathbf{f}$ w.r.t. $\overrightarrow{\mathbf{r}}$ and $\overrightarrow{\mathbf{q}}$ :

$$
\mathbf{K}(\overrightarrow{\mathbf{r}}, \overrightarrow{\mathbf{q}}):=\frac{d \mathbf{f}}{d(\overrightarrow{\mathbf{r}}, \overrightarrow{\mathbf{q}})}=\left(\begin{array}{ll}
\frac{d \mathbf{f}_{\mathbf{n}}\left(\tau_{i}\right)}{d \mathbf{r}_{j}} & \frac{d \mathbf{f}_{\mathbf{n}}\left(\tau_{i}\right)}{d \mathbf{q}_{j}} \\
\frac{d \mathbf{f}_{\mathbf{m}}\left(\tau_{i}\right)}{d \mathbf{r}_{j}} & \frac{d \mathbf{f}_{\mathbf{m}}\left(\tau_{i}\right)}{d \mathbf{q}_{j}} \\
\frac{d \mathbf{f}_{\mathbf{q}}\left(\tau_{i}\right)}{d \mathbf{r}_{j}} & \frac{d \mathbf{q}_{\mathbf{q}}\left(\tau_{i}\right)}{d \mathbf{q}_{j}}
\end{array}\right)_{i, j=1, \ldots, n} .
$$

We derive the individual components of the tangential stiffness matrix $\mathbf{K}$ in detail, since they are required for the implementation of the method. For internal collocation points $i=2, \ldots, n-1$ they are:

$$
\begin{aligned}
\frac{d \mathbf{f}_{\mathbf{n}}\left(\tau_{i}\right)}{d \mathbf{r}_{j}} & =\left(\mathbf{R}^{\prime} \mathbf{C} \mathbf{R}^{\top}+\mathbf{R} \mathbf{C} \mathbf{R}^{\prime \top}\right) N_{j}^{\prime}+\mathbf{R} \mathbf{C} \mathbf{R}^{\top} N_{j}^{\prime \prime}, \\
\frac{d \mathbf{f}_{\mathbf{n}}\left(\tau_{i}\right)}{d \mathbf{q}_{j}} & =\frac{d \mathbf{R}^{\prime}}{d \mathbf{q}_{j}}(\boldsymbol{\sigma})+\frac{d \mathbf{R}}{d \mathbf{q}_{j}}\left(\boldsymbol{\sigma}^{\prime}\right)+\mathbf{R}^{\prime} \mathbf{C} \frac{d \mathbf{R}^{\top}}{d \mathbf{q}_{j}}\left(\mathbf{r}_{h}^{\prime}\right)+\mathbf{R C}\left(\frac{d \mathbf{R}^{\prime \top}}{d \mathbf{q}_{j}}\left(\mathbf{r}_{h}^{\prime}\right)+\frac{d \mathbf{R}^{\top}}{d \mathbf{q}_{j}}\left(\mathbf{r}_{h}^{\prime \prime}\right)\right), \\
\frac{d \mathbf{f}_{\mathbf{m}}\left(\tau_{i}\right)}{d \mathbf{r}_{j}} & =[\mathbf{n}]_{\times} N_{j}^{\prime}-\left[\mathbf{r}_{h}^{\prime}\right]_{\times} \mathbf{R} \mathbf{C} \mathbf{R}^{\top} N_{j}^{\prime}, \\
\frac{d \mathbf{f}_{\mathbf{m}}\left(\tau_{i}\right)}{d \mathbf{q}_{j}} & =\frac{d \mathbf{R}^{\prime}}{d \mathbf{q}_{j}}(\boldsymbol{\chi})+\frac{d \mathbf{R}}{d \mathbf{q}_{j}}\left(\chi^{\prime}\right)+\mathbf{R}^{\prime} \mathbf{D} \frac{d \boldsymbol{\kappa}}{d \mathbf{q}_{j}}+\mathbf{R D} \frac{d \boldsymbol{\kappa}^{\prime}}{d \mathbf{q}_{j}}-\left[\mathbf{r}_{h}^{\prime}\right]_{\times}\left(\frac{d \mathbf{R}}{d \mathbf{q}_{j}}(\boldsymbol{\sigma})+\mathbf{R C} \frac{d \mathbf{R}^{\top}}{d \mathbf{q}_{j}}\left(\mathbf{r}_{h}^{\prime}\right)\right), \\
\frac{d \mathbf{f}_{\mathbf{q}}\left(\tau_{i}\right)}{d \mathbf{r}_{j}} & =\mathbf{0}_{1 \times 3}, \\
\frac{d \mathbf{f}_{\mathbf{q}}\left(\tau_{i}\right)}{d \mathbf{q}_{j}} & =2 \mathbf{q}_{h}^{\top} N_{j} .
\end{aligned}
$$

For boundary collocation points $i=1, n$ they are:

$$
\begin{aligned}
\frac{d \mathbf{f}_{\mathbf{n}}\left(\tau_{i}\right)}{d \mathbf{r}_{j}} & =\mathbf{R C} \mathbf{R}^{\top} N_{j}^{\prime}, & \frac{d \mathbf{f}_{\mathbf{n}}\left(\tau_{i}\right)}{d \mathbf{q}_{j}} & =\frac{d \mathbf{R}}{d \mathbf{q}_{j}}(\boldsymbol{\sigma})+\mathbf{R C} \frac{d \mathbf{R}^{\top}}{d \mathbf{q}_{j}}\left(\mathbf{r}_{h}^{\prime}\right), \\
\frac{d \mathbf{f}_{\mathbf{m}}\left(\tau_{i}\right)}{d \mathbf{r}_{j}} & =\mathbf{0}_{3 \times 3}, & \frac{d \mathbf{f}_{\mathbf{m}}\left(\tau_{i}\right)}{d \mathbf{q}_{j}} & =\frac{d \mathbf{R}}{d \mathbf{q}_{j}}(\boldsymbol{\chi})+\mathbf{R D} \frac{d \boldsymbol{\kappa}}{d \mathbf{q}_{j}}, \\
\frac{d \mathbf{f}_{\mathbf{q}}\left(\tau_{i}\right)}{d \mathbf{r}_{j}} & =\mathbf{0}_{1 \times 3}, & \frac{d \mathbf{f}_{\mathbf{q}}\left(\tau_{i}\right)}{d \mathbf{q}_{j}} & =2 \mathbf{q}_{h}^{\top} N_{j} .
\end{aligned}
$$

Above all quantities refer to their evaluations at $\tau_{i}$, e.g. $\mathbf{r}_{h}$ represents $\mathbf{r}_{h}\left(\tau_{i}\right), \mathbf{n}$ stands for $\mathbf{n}\left(\tau_{i}\right), N_{j}$ for $N_{j}\left(\tau_{i}\right)$ etc.

Defining the matrices

$$
\begin{aligned}
& \mathbf{D}_{1}(\mathbf{q})=\frac{d \mathbf{d}_{1}(\mathbf{q})}{d \mathbf{q}}=2\left(\begin{array}{cccc}
q_{1} & -q_{2} & -q_{3} & q_{4} \\
q_{2} & q_{1} & q_{4} & q_{3} \\
q_{3} & -q_{4} & q_{1} & -q_{2}
\end{array}\right), \\
& \mathbf{D}_{2}(\mathbf{q})=\frac{d \mathbf{d}_{2}(\mathbf{q})}{d \mathbf{q}}=2\left(\begin{array}{cccc}
q_{2} & q_{1} & -q_{4} & -q_{3} \\
-q_{1} & q_{2} & -q_{3} & q_{4} \\
q_{4} & q_{3} & q_{2} & q_{1}
\end{array}\right), \\
& \mathbf{D}_{3}(\mathbf{q})=\frac{d \mathbf{d}_{3}(\mathbf{q})}{d \mathbf{q}}=2\left(\begin{array}{cccc}
q_{3} & q_{4} & q_{1} & q_{2} \\
-q_{4} & q_{3} & q_{2} & -q_{1} \\
-q_{1} & -q_{2} & q_{3} & q_{4}
\end{array}\right)
\end{aligned}
$$


as the derivatives of the column vector representation $\mathbf{R}=\left(\mathbf{d}_{1}, \mathbf{d}_{2}, \mathbf{d}_{3}\right)$ as defined in $(6)$ and $(8)$ w.r.t. $\mathbf{q}$, we can evaluate the arc-length derivatives of $\mathbf{R}$ as:

$$
\begin{aligned}
& \mathbf{R}^{\prime}(\mathbf{q})=\frac{d \mathbf{R}(\mathbf{q})}{d s}=\left(\mathbf{d}_{1}^{\prime}, \mathbf{d}_{2}^{\prime}, \mathbf{d}_{3}^{\prime}\right)(\mathbf{q}) \quad \text { with } \mathbf{d}_{k}^{\prime}=\mathbf{D}_{k}(\mathbf{q}) \mathbf{q}^{\prime}, k=1,2,3 \\
& \mathbf{R}^{\prime \prime}(\mathbf{q})=\frac{d^{2} \mathbf{R}(\mathbf{q})}{d s^{2}}=\left(\mathbf{d}_{1}^{\prime \prime}, \mathbf{d}_{2}^{\prime \prime}, \mathbf{d}_{3}^{\prime \prime}\right)(\mathbf{q}) \quad \text { with } \mathbf{d}_{k}^{\prime \prime}=\mathbf{D}_{k}\left(\mathbf{q}^{\prime}\right) \mathbf{q}^{\prime}+\mathbf{D}_{k}(\mathbf{q}) \mathbf{q}^{\prime \prime}, k=1,2,3 .
\end{aligned}
$$

Since $d \mathbf{R} / d \mathbf{q}_{j}, d \mathbf{R}^{\prime} / d \mathbf{q}_{j}$ and $d \mathbf{R}^{\prime \prime} / d \mathbf{q}_{j}$ are $3 \times 4 \times 3$-tensors, we provide their derivatives now in terms of the column vectors $\mathbf{d}_{k}, k=1,2,3$ :

$$
\begin{gathered}
\frac{d \mathbf{d}_{k}}{d \mathbf{q}_{j}}\left(\tau_{i}\right)=\mathbf{D}_{k}\left(\mathbf{q}\left(\tau_{i}\right)\right) N_{j}, \quad \frac{d \mathbf{d}_{k}^{\prime}}{d \mathbf{q}_{j}}\left(\tau_{i}\right)=\mathbf{D}_{k}\left(\mathbf{q}^{\prime}\left(\tau_{i}\right)\right) N_{j}+\mathbf{D}_{k}\left(\mathbf{q}\left(\tau_{i}\right)\right) N_{j}^{\prime} \\
\frac{d \mathbf{d}_{k}^{\prime \prime}}{d \mathbf{q}_{j}}\left(\tau_{i}\right)=\mathbf{D}_{k}\left(\mathbf{q}^{\prime \prime}\left(\tau_{i}\right)\right) N_{j}+2 \mathbf{D}_{k}\left(\mathbf{q}^{\prime}\left(\tau_{i}\right)\right) N_{j}^{\prime}+\mathbf{D}_{k}\left(\mathbf{q}\left(\tau_{i}\right)\right) N_{j}^{\prime \prime}
\end{gathered}
$$

Thus, multiplication of for instance $d \mathbf{R} / d \mathbf{q}_{j}$ on a vector $\mathbf{y} \in \mathbb{R}^{3}$, as used in (39), results in:

$$
\frac{d \mathbf{R}}{d \mathbf{q}_{j}}(\mathbf{y})=\frac{d \mathbf{d}_{1}}{d \mathbf{q}_{j}} y_{1}+\frac{d \mathbf{d}_{2}}{d \mathbf{q}_{j}} y_{2}+\frac{d \mathbf{d}_{3}}{d \mathbf{q}_{j}} y_{3} .
$$

Furthermore, we still need to specify the derivatives of $\boldsymbol{\kappa}$ and $\boldsymbol{\kappa}^{\prime}$ w.r.t. $\mathbf{q}_{j}$ :

$$
\frac{d \boldsymbol{\kappa}}{d \mathbf{q}_{j}}=\left(\begin{array}{l}
\mathbf{d}_{2}^{\prime \top} \frac{d \mathbf{d}_{3}}{d \mathbf{q}_{j}}+\mathbf{d}_{3}^{\top} \frac{d \mathbf{d}_{2}}{d \mathbf{q}_{j}} \\
\mathbf{d}_{3}^{\prime \top} \frac{d \mathbf{d}_{1}}{d \mathbf{q}_{j}}+\mathbf{d}_{1}^{\top} \frac{d \mathbf{d}_{3}}{d \mathbf{q}_{j}} \\
\mathbf{d}_{1}^{\prime \top} \frac{d \mathbf{d}_{2}}{d \mathbf{q}_{j}}+\mathbf{d}_{2}^{\top} \frac{d \mathbf{d}_{1}}{d \mathbf{q}_{j}}
\end{array}\right), \quad \frac{d \boldsymbol{\kappa}^{\prime}}{d \mathbf{q}_{j}}=\left(\begin{array}{l}
\mathbf{d}_{2}^{\prime \prime \top} \frac{d \mathbf{d}_{3}}{d \mathbf{q}}+\mathbf{d}_{2}^{\prime \top} \frac{d \mathbf{d}_{3}^{\prime}}{d \mathbf{q}_{j}}+\mathbf{d}_{3}^{\top} \frac{d \mathbf{d}_{2}^{\prime \prime}}{d \mathbf{q}_{j}}+\mathbf{d}_{3}^{\prime \top} \frac{d \mathbf{d}_{2}^{\prime}}{d \mathbf{q}_{j}} \\
\mathbf{d}_{3}^{\prime \prime \top} \frac{d \mathbf{d}_{1}}{d \mathbf{q}}+\mathbf{d}_{3}^{\prime \top} \frac{d \mathbf{d}_{1}^{\prime}}{d \mathbf{q}_{j}}+\mathbf{d}_{1}^{\top} \frac{d \mathbf{d}_{3}^{\prime \prime}}{d \mathbf{q}_{j}}+\mathbf{d}_{1}^{\prime \top} \frac{d \mathbf{d}_{3}^{\prime}}{d \mathbf{q}_{j}} \\
\mathbf{d}_{1}^{\prime \prime \top} \frac{d \mathbf{d}_{2}}{d \mathbf{q}}+\mathbf{d}_{1}^{\prime \top} \frac{d \mathbf{d}_{2}^{\prime}}{d \mathbf{q}_{j}}+\mathbf{d}_{2}^{\top} \frac{d \mathbf{d}_{1}^{\prime \prime}}{d \mathbf{q}_{j}}+\mathbf{d}_{2}^{\prime \top} \frac{d \mathbf{d}_{1}^{\prime}}{d \mathbf{q}_{j}}
\end{array}\right) .
$$

\section{Coupling of rods and rod structures}

Having introduced the Cosserat model and isogeometric collocation method for the modeling and simulation of a single rod, we now want to extend the framework to rod structures, i.e. meshes of interconnected, coupled rods. Therefore we need to define constraints for the continuity of displacements and rotations, and the transfer of forces and moments at the coupling points or interfaces. Similar to [53], we are going to derive a rigid, quasi- $G^{1}$-continuous coupling of the rods. In 30 it is already described how the collocation equations have to be adapted for coupling of multiple patches in 2D and 3D isogeometric collocation of linear elasticity, and here we transfer the approach to the collocation of Cosserat rods.

Without loss of generality we can assume that rods can only be connected to other rods at their end points, since otherwise a single rod could simply be split into two parts. Furthermore, we assume that $k \geq 2 \operatorname{rods}\left(\mathbf{r}^{(i)}, \mathbf{R}^{(i)}\right), i=1, \ldots, k$ intersect in a given interface and that for each rod the end control point at the interface is denoted by index $e_{i} \in\left\{1, n^{(i)}\right\}$ and the end collocation parameter of the NURBS parameterization by $\tilde{\tau}_{i} \in\{0,1\}$.

The rods have to remain connected under deformation, which means that the centerlines must be continuous at the interface:

$$
\mathbf{r}_{h}^{(1)}\left(\tilde{\tau}_{1}\right)=\ldots=\mathbf{r}_{h}^{(k)}\left(\tilde{\tau}_{k}\right) .
$$

Due to the endpoint interpolation property of B-Spline and NURBS curves with open knot vectors, it follows that the end centerline control points must be equal:

$$
\mathbf{r}_{e_{1}}^{(1)}=\ldots=\mathbf{r}_{e_{k}}^{(k)}
$$

Furthermore, for a rigid coupling it is necessary that the change of orientation of the frames from the initial to the deformed configuration must be the same for all rods at the interface. In terms of the rotation matrices this can be formulated as

$$
\mathbf{R}^{(1)}\left(\tilde{\tau}_{1}\right) \mathbf{R}_{0}^{(1)}\left(\tilde{\tau}_{1}\right)^{-1}=\ldots=\mathbf{R}^{(k)}\left(\tilde{\tau}_{k}\right) \mathbf{R}_{0}^{(k)}\left(\tilde{\tau}_{k}\right)^{-1}
$$




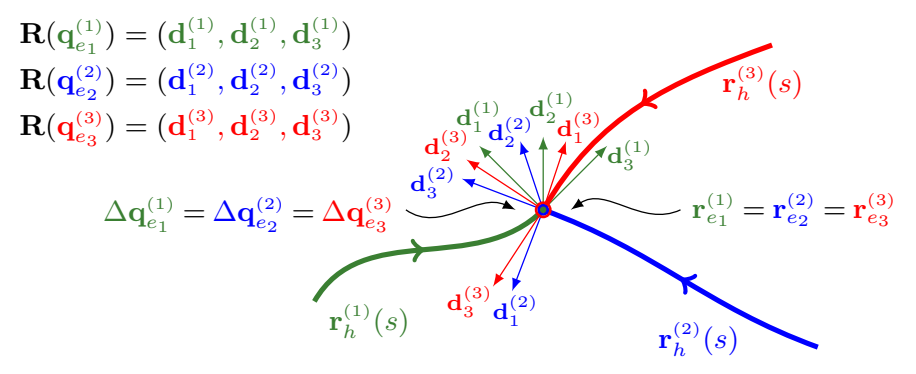

Constraint equations:

$$
\begin{aligned}
& \mathbf{f}_{\mathrm{n}}^{(1)}\left(\tilde{\tau}_{1}\right)+\mathbf{f}_{\mathrm{n}}^{(2)}\left(\tilde{\tau}_{2}\right)+\mathbf{f}_{\mathrm{n}}^{(3)}\left(\tilde{\tau}_{3}\right)=\mathbf{0}, \\
& \mathbf{r}_{e_{1}}^{(1)}-\mathbf{r}_{e_{3}}^{(3)}=\mathbf{0}, \\
& \mathbf{r}_{e_{1}}^{(1)}-\mathbf{r}_{e_{2}}^{(2)}=\mathbf{0}, \\
& \mathbf{f}_{\mathrm{m}}^{(1)}\left(\tilde{\tau}_{1}\right)+\mathbf{f}_{\mathrm{m}}^{(2)}\left(\tilde{\tau}_{2}\right)+\mathbf{f}_{\mathrm{m}}^{(3)}\left(\tilde{\tau}_{3}\right)=\mathbf{0}, \\
& \mathbf{q}_{e_{1}}^{(1)} \overline{\mathbf{q}}_{0, e_{1}}^{(1)}-\mathbf{q}_{e_{2}}^{(2)} \overline{\mathbf{q}}_{0, e_{2}}^{(2)}=\mathbf{0}, \\
& \mathbf{q}_{e_{1}}^{(1)} \overline{\mathbf{q}}_{0, e_{1}}^{(1)}-\mathbf{q}_{e_{3}}^{(3)} \overline{\mathbf{q}}_{0, e_{3}}^{(3)}=\mathbf{0} .
\end{aligned}
$$

Figure 3: Illustration of rod coupling, with three rods connected in a common interface

since the current rotation can be expressed as $\mathbf{R}=\Delta \mathbf{R} \mathbf{R}_{0}$, where $\Delta \mathbf{R}$ is the change of rotation resp. orientation from the initial state $\mathbf{R}_{0}$. This change of rotation can be expressed as $\Delta \mathbf{q}=\mathbf{q} \overline{\mathbf{q}}_{0}$ and leads to the following condition in terms of the end quaternion control points at the interface:

$$
\mathbf{q}_{e_{1}}^{(1)} \overline{\mathbf{q}}_{0, e_{1}}^{(1)}=\ldots=\mathbf{q}_{e_{k}}^{(k)} \overline{\mathbf{q}}_{0, e_{k}}^{(k)},
$$

where ${ }^{-}$denotes conjugation of the quaternion.

Furthermore, the equilibria of linear and angular momentum need to be consistent at an interface as well. As in [30, we thus have to sum up the contributions of each individual rod from (35) in a single collocation equation:

$$
\sum_{i=1}^{k} I\left(e_{i}\right) \mathbf{f}_{\mathrm{n}}^{(i)}\left(\tilde{\tau}_{i}\right)=\mathbf{0}, \quad \sum_{i=1}^{k} I\left(e_{i}\right) \mathbf{f}_{\mathrm{m}}^{(i)}\left(\tilde{\tau}_{i}\right)=\mathbf{0} .
$$

Here $I\left(e_{k}\right) \in\{-1,+1\}$ is an orientation factor which is +1 if $e_{k}$ is the first and -1 if $e_{k}$ is the last control point of the rod - analogous to the concept of outer normals in [30].

Finally, a global force vector $\mathbf{f}$ can be assembled from the independent contributions of individual rods $\mathbf{f}^{(i)}$ from (35). Then the interface conditions derived above have to be applied. Thus for each interface of $k$ rods, the equations corresponding to collocation point $\tilde{\tau}_{1}$ are replaced by the accumulated contributions from (50) and for $\tilde{\tau}_{2}, \ldots, \tilde{\tau}_{k}$ by the $k-1$ linear conditions arising from (47) and 49), while the quaternion normalization conditions from 35 remain unchanged and have to be enforced individually:

$$
\begin{aligned}
& i=1: \quad\left\{\begin{array}{l}
\mathbf{f}_{\mathrm{n}}^{(1)}\left(\tilde{\tau}_{1}\right) \leftarrow \sum_{j=1}^{k} I\left(e_{j}\right) \mathbf{f}_{\mathrm{n}}^{(j)}\left(\tilde{\tau}_{j}\right), \\
\mathbf{f}_{\mathrm{m}}^{(1)}\left(\tilde{\tau}_{1}\right) \leftarrow \sum_{j=1}^{k} I\left(e_{j}\right) \mathbf{f}_{\mathrm{m}}^{(j)}\left(\tilde{\tau}_{j}\right), \\
\mathbf{f}_{\mathrm{q}}^{(1)}\left(\tilde{\tau}_{1}\right)=\mathbf{q}_{h}\left(\tilde{\tau}_{1}\right)^{\top} \mathbf{q}_{h}\left(\tilde{\tau}_{1}\right)-1,
\end{array}\right. \\
& i=2, \ldots, k:\left\{\begin{array}{l}
\mathbf{f}_{\mathrm{n}}^{(i)}\left(\tilde{\tau}_{i}\right) \leftarrow \mathbf{r}_{e_{1}}^{(1)}-\mathbf{r}_{e_{i}}^{(i)}, \\
\mathbf{f}_{\mathrm{m}}^{(i)}\left(\tilde{\tau}_{i}\right) \leftarrow \mathbf{q}_{e_{1}}^{(1)} \overline{\mathbf{q}}_{0, e_{1}}^{(1)}-\mathbf{q}_{e_{i}}^{(i)} \overline{\mathbf{q}}_{0, e_{i}}^{(i)}, \\
\mathbf{f}_{\mathbf{q}}^{(i)}\left(\tilde{\tau}_{i}\right)=\mathbf{q}_{h}\left(\tilde{\tau}_{i}\right)^{\top} \mathbf{q}_{h}\left(\tilde{\tau}_{i}\right)-1 .
\end{array}\right.
\end{aligned}
$$

The coupling of three rods in a common interface and associated collocation equations are also illustrated in Fig. 3 .

Of course, for the linearization of the global force vector the same procedure has to be applied also to the affected rows of the global tangential stiffness matrix $\mathbf{K}$, which has to be assembled from individual rod contributions $\mathbf{K}^{(i)}$ as given in 38 .

Since we enforce the continuity of centerline positions resp. displacements and change of cross-sections orientations in terms of the rotation quaternions, no deformation and relative change of rotation of the rods with respect to each other is possible at the interface - which is why we refer to it as "rigid" coupling. Furthermore, when the centerline tangents and cross-section orientations of two rods match in the initial 
configuration, they are initially $G^{1}$-continuous at the interface. Since this property is preserved throughout the deformation by the presented coupling method, i.e. the curvatures (which depend on the derivatives of rotations) remain continuous at the interface, we also call the method "quasi- $G^{1}$-continuous".

\section{Mixed isogeometric collocation method}

It is a well-known phenomenon that beam or rod discretizations may suffer from shear locking, which means that for decreasing thickness of a beam (cross-section diameter of a rod), the accuracy and convergence of the numerical discretization method can deteriorate. This behavior was already observed for the isogeometric collocation of the linear Timoshenko beam and rod models in [32, 33. To alleviate the locking phenomenon, a mixed collocation method was developed and investigated theoretically and numerically in [32, 33. In the following, we transfer the approach to the isogeometric collocation of Cosserat rods.

In addition to the independent field variables for centerline positions $\mathbf{r}_{h}$ and rotation quaternions $\mathbf{q}_{h}$ (33), we now also discretize the internal forces and moments as independent NURBS curves:

$$
\begin{aligned}
\mathbf{n}_{h}:[0,1] \rightarrow \mathbb{R}^{3}, & \mathbf{n}_{h}(t)=\sum_{i=1}^{n} N_{i}(t) \mathbf{n}_{i} \\
\mathbf{m}_{h}:[0,1] \rightarrow \mathbb{R}^{3}, & \mathbf{m}_{h}(t)=\sum_{i=1}^{n} N_{i}(t) \mathbf{m}_{i} .
\end{aligned}
$$

In 33 it was shown that mixed collocation methods for linear Timoshenko rods are shear locking-free for any combination of degrees of basis functions for the discretization spaces of independent fields. Based on this analysis, we expect the same property to hold also in the present nonlinear Cosserat rod problem and use the same basis functions $N_{i}$ of degree $p$ for all independent variables.

Furthermore, numerical discretizations for initially curved rods may also suffer from so-called membrane locking. This phenomenon was intensively investigated for isogeometric finite element discretizations of the Timoshenko beam problem in [20. Since the mixed formulation presented here is based on an independent discretization of both internal forces and moments, see (52), it should alleviate the shear locking problem as well.

The collocated equilibrium equations can now be formulated in terms of the newly introduced independent fields $\mathbf{n}_{h}$ and $\mathbf{m}_{h}$. Furthermore, two additional equations have to be added to relate them to $\mathbf{r}_{h}$ and $\mathbf{q}_{h}$. Here these are the relations of the stresses to the internal forces and moments as given in (18). Altogether, the new set of collocated equations at internal collocation points $\tau_{i}, i=2, \ldots, n-1$ reads:

$$
\begin{array}{rlrl}
\mathbf{f}_{\mathbf{n}}\left(\tau_{i}\right) & =\mathbf{n}_{h}^{\prime}\left(\tau_{i}\right)+\hat{\mathbf{n}}\left(\tau_{i}\right) & =\mathbf{0}, \\
\mathbf{f}_{\mathbf{m}}\left(\tau_{i}\right) & =\mathbf{m}_{h}^{\prime}\left(\tau_{i}\right)+\mathbf{r}_{h}^{\prime}\left(\tau_{i}\right) \times \mathbf{n}_{h}\left(\tau_{i}\right)+\hat{\mathbf{m}}\left(\tau_{i}\right) & & =\mathbf{0}, \\
\mathbf{f}_{\mathbf{q}}\left(\tau_{i}\right) & =\mathbf{q}_{h}\left(\tau_{i}\right)^{\top} \mathbf{q}_{h}\left(\tau_{i}\right)-1 & & =0, \\
\mathbf{f}_{\mathbf{u}}\left(\tau_{i}\right) & =\mathbf{n}_{h}\left(\tau_{i}\right)-(\mathbf{R} \boldsymbol{\sigma})\left(\tau_{i}\right) & & =\mathbf{0}, \\
\mathbf{f}_{\mathbf{v}}\left(\tau_{i}\right) & =\mathbf{m}_{h}\left(\tau_{i}\right)-(\mathbf{R} \boldsymbol{\chi})\left(\tau_{i}\right) & & =\mathbf{0} .
\end{array}
$$

At the Neumann boundaries the collocated equations for $\tau_{1}=0$ and $\tau_{n}=1$ are consequently:

$$
\begin{array}{rll}
\mathbf{f}_{\mathbf{n}}\left(\tau_{i}\right) & =\mathbf{n}_{h}\left(\tau_{i}\right)-\overline{\mathbf{n}}\left(\tau_{i}\right) & =\mathbf{0}, \\
\mathbf{f}_{\mathbf{m}}\left(\tau_{i}\right) & =\mathbf{m}_{h}\left(\tau_{i}\right)-\overline{\mathbf{m}}\left(\tau_{i}\right) & =\mathbf{0}, \\
\mathbf{f}_{\mathbf{q}}\left(\tau_{i}\right) & =\mathbf{q}_{h}\left(\tau_{i}\right)^{\top} \mathbf{q}_{h}\left(\tau_{i}\right)-1 & =0, \\
\mathbf{f}_{\mathbf{u}}\left(\tau_{i}\right) & =\mathbf{n}_{h}\left(\tau_{i}\right)-(\mathbf{R} \boldsymbol{\sigma})\left(\tau_{i}\right) & =\mathbf{0}, \\
\mathbf{f}_{\mathbf{v}}\left(\tau_{i}\right) & =\mathbf{m}_{h}\left(\tau_{i}\right)-(\mathbf{R} \boldsymbol{\chi})\left(\tau_{i}\right) & =\mathbf{0} .
\end{array}
$$




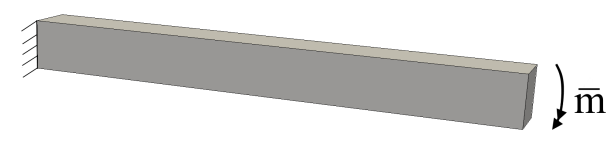

(a) initially straight cantilever

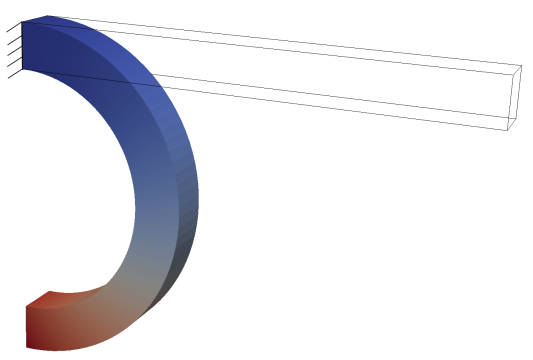

(b) deformed cantilever

Figure 4: Bending of straight cantilever; an end moment of magnitude $\bar{m}=\pi E I$ is applied to the initially straight cantilever beam of length $L=1$ (a) and leads to bending into a semi-circle of radius $1 / \pi(\mathrm{b})$

For this mixed method, (53) and (54) define a set of $13 \cdot n$ nonlinear equations for the $3 \cdot n$ unknown control points of $\mathbf{r}_{h}, 4 \cdot n$ unknowns of $\mathbf{q}_{h}$, and now additionally $3 \cdot n$ unknowns of both $\mathbf{n}_{h}$ and $\mathbf{m}_{h}$ :

$$
\mathbf{f}: \mathbb{R}^{3 \cdot n} \times \mathbb{R}^{4 \cdot n} \times \mathbb{R}^{3 \cdot n} \times \mathbb{R}^{3 \cdot n} \rightarrow \mathbb{R}^{13 \cdot n}: \quad \mathbf{f}(\overrightarrow{\mathbf{r}}, \overrightarrow{\mathbf{q}}, \overrightarrow{\mathbf{n}}, \overrightarrow{\mathbf{m}})=\left(\begin{array}{c}
\mathbf{f}_{\mathrm{n}}\left(\tau_{i}\right) \\
\mathbf{f}_{\mathrm{m}}\left(\tau_{i}\right) \\
\mathbf{f}_{\mathrm{q}}\left(\tau_{i}\right) \\
\mathbf{f}_{\mathrm{u}}\left(\tau_{i}\right) \\
\mathbf{f}_{\mathrm{v}}\left(\tau_{i}\right)
\end{array}\right)_{i=1, \ldots, n} \quad(\overrightarrow{\mathbf{r}}, \overrightarrow{\mathbf{q}}, \overrightarrow{\mathbf{n}}, \overrightarrow{\mathbf{m}})=\mathbf{0}
$$

where the control points of $\mathbf{n}_{h}$ and $\mathbf{m}_{h}$ are written as vectors $\overrightarrow{\mathbf{n}}=\left(\mathbf{n}_{i}\right)_{i=1, \ldots, n} \in \mathbb{R}^{3 \cdot n}$ and $\overrightarrow{\mathbf{m}}=\left(\mathbf{m}_{i}\right)_{i=1, \ldots, n} \in$ $\mathbb{R}^{3 \cdot n}$.

The solution of (55) using a Newton's method and the evaluation of its derivatives is simpler than for (36), since only first derivatives w.r.t. the unknown control point vectors are required. This also releases regularity requirements on the basis functions $N_{i}$, which now only need to be in $C^{1}[0,1]$ instead of $C^{2}[0,1]$ as before.

The coupling of Cosserat rods with mixed discretization is straight-forward and can be done exactly as presented in Sect. 5, since consistent centerline deformation, change of rotation, as well as equilibria of internal forces and moments are enforced by the described coupling conditions. This means that equations $(54)_{1}$ and $(54)_{2}$ have to be modified in the same way as presented in $(51)$, while $(54)_{3},(54)_{4}$ and $(54)_{5}$ remain unchanged and have to be enforced independently.

\section{Numerical applications}

With a number of numerical applications we now want to verify the isogeometric collocation method for the Cosserat rod model, investigate its numerical properties and apply it to the simulation of complex deformations of rods and rod structures.

\subsection{Bending of straight cantilever}

In our first numerical example we want to study the convergence properties of the proposed isogeometric collocation methods for the Cosserat rod. We apply our method to the pure bending of a straight cantilever beam by an end moment. This problem is a typical benchmark for nonlinear beam formulations and has an analytical solution, since the application of an end moment $\bar{m}=\phi E I_{1}$ to a straight cantilever beam of unit length $L=1$ results in a uniform bending into a circular arc of angle $\phi$ and radius $1 / \phi$ with constant curvature $\kappa_{1}=\phi$, see Fig. 4. Here we use a rod with quadratic cross-section of thickness $t=b=h$, Young's modulus $E=1 \cdot 10^{9}$, Poisson's ratio $\nu=0.5$, and $k_{1}=k_{2}=5 / 6$, see (17). The cross-section area is then $A=t^{2}$ and the 2 nd moment of area is $I_{1}=t^{4} / 12$. For decreasing value of the thickness $t$, locking of the 


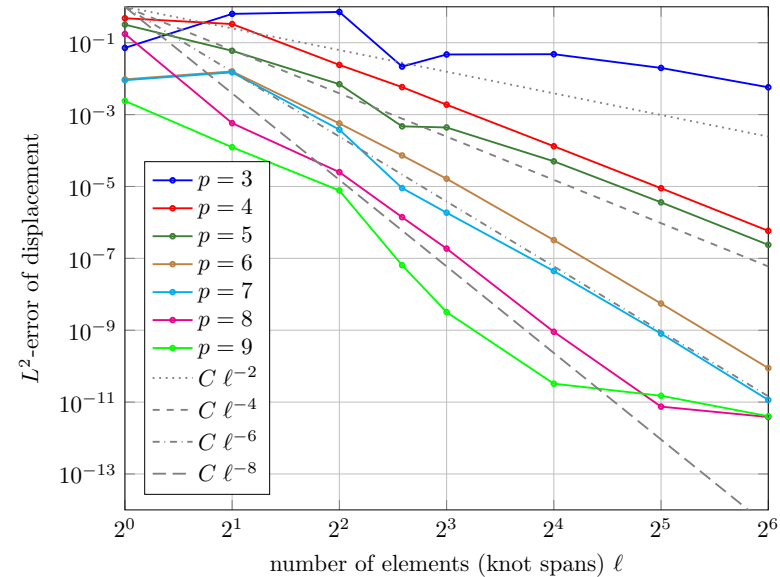

(a) $t=0.1$ : primal formulation $\left(\mathbf{r}_{h}, \mathbf{q}_{h}\right)$

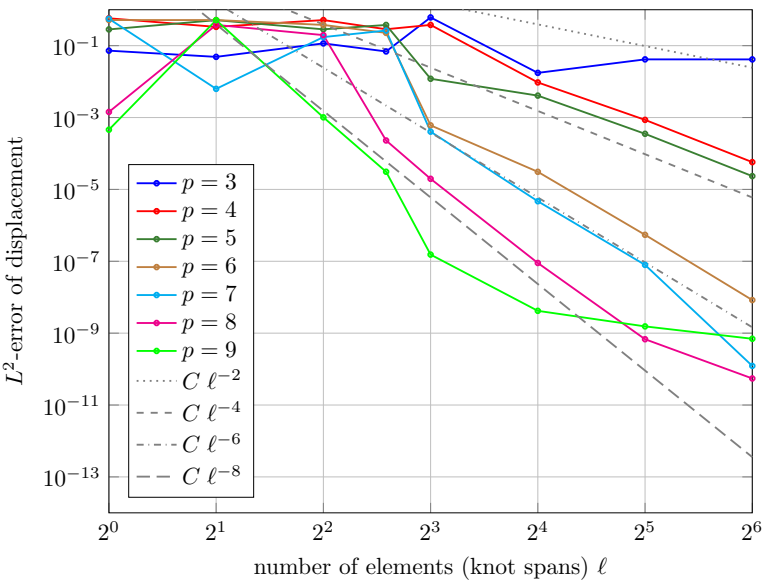

(c) $t=0.01:$ primal formulation $\left(\mathbf{r}_{h}, \mathbf{q}_{h}\right)$

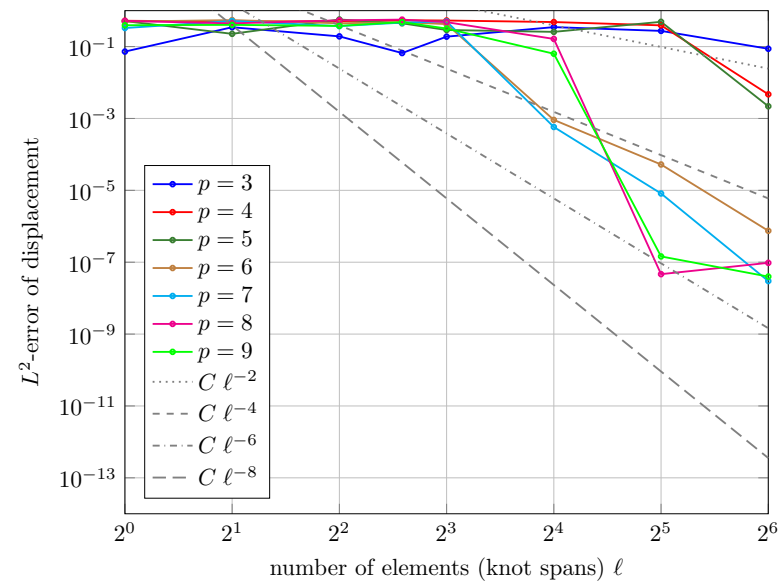

(e) $t=0.001:$ primal formulation $\left(\mathbf{r}_{h}, \mathbf{q}_{h}\right)$

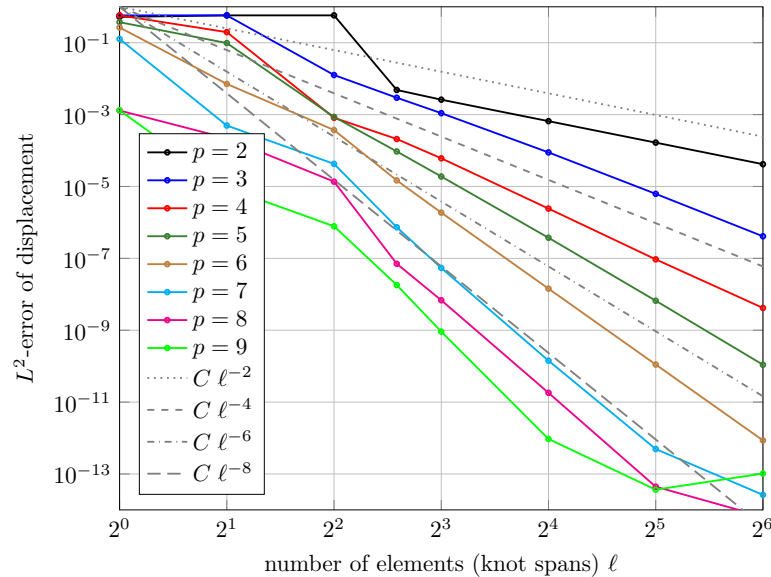

(b) $t=0.1:$ mixed formulation $\left(\mathbf{r}_{h}, \mathbf{q}_{h}, \mathbf{n}_{h}, \mathbf{m}_{h}\right)$

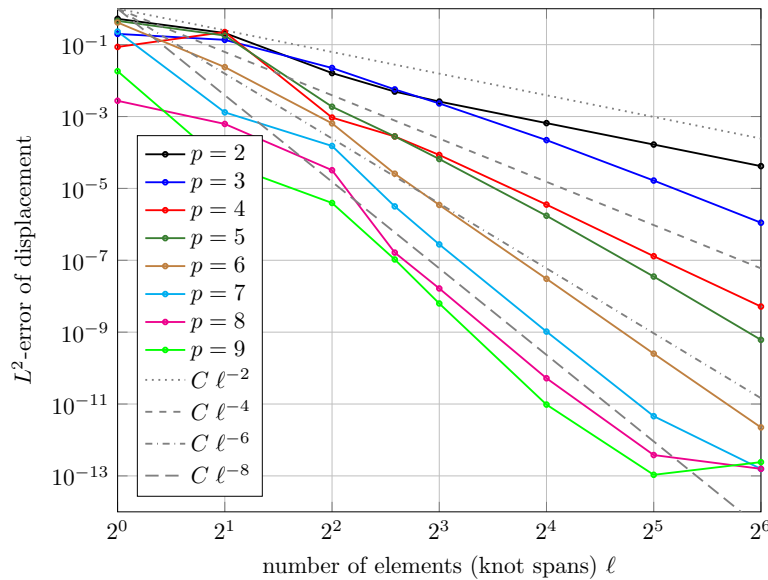

(d) $t=0.01$ : mixed formulation $\left(\mathbf{r}_{h}, \mathbf{q}_{h}, \mathbf{n}_{h}, \mathbf{m}_{h}\right)$

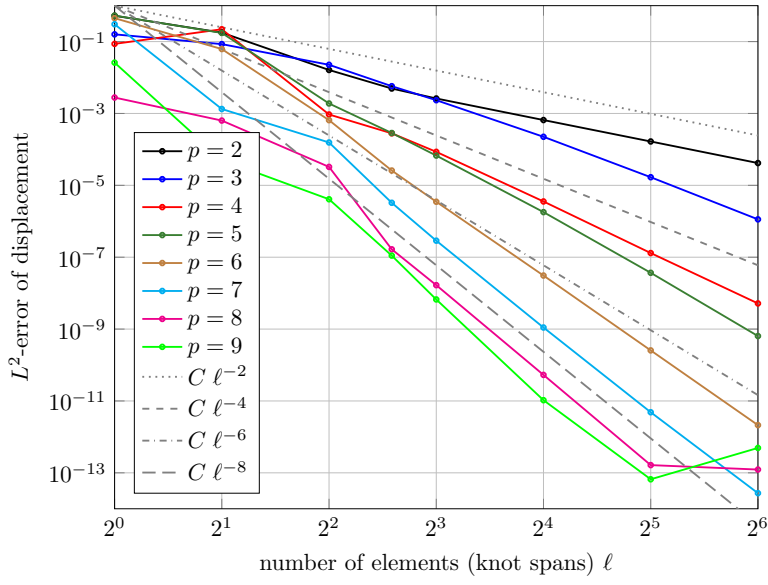

(f) $t=0.001:$ mixed formulation $\left(\mathbf{r}_{h}, \mathbf{q}_{h}, \mathbf{n}_{h}, \mathbf{m}_{h}\right)$

Figure 5: Bending of straight cantilever; investigation of locking for thickness parameter $t=0.1,0.01,0.001$ by comparison of convergence of $L^{2}$-error of displacement of centerline $\left\|\mathbf{r}^{h}-\mathbf{r}^{e}\right\|_{L^{2}}$ for NURBS with degree $p=(2) 3,, \ldots, 9$ and $\ell=1-64$ elements for primal and mixed formulations 


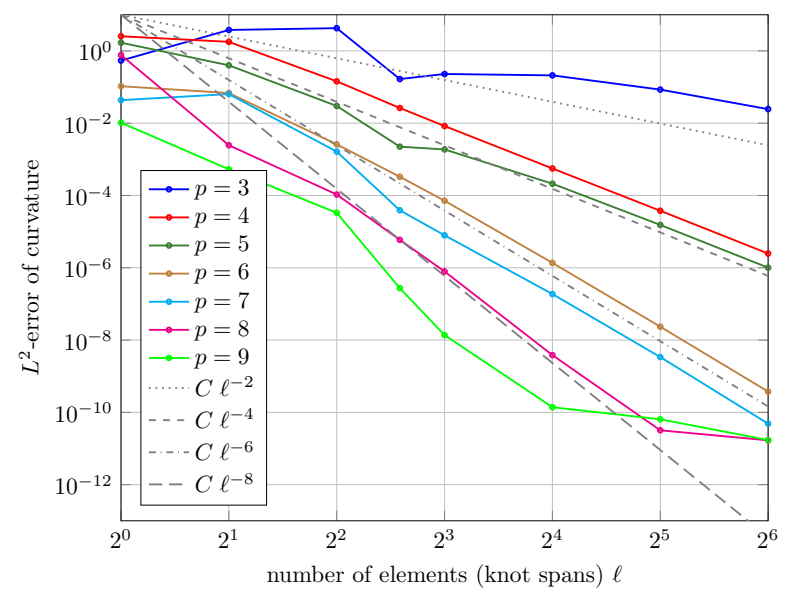

(a) $t=0.1$ : primal formulation $\left(\mathbf{r}_{h}, \mathbf{q}_{h}\right)$

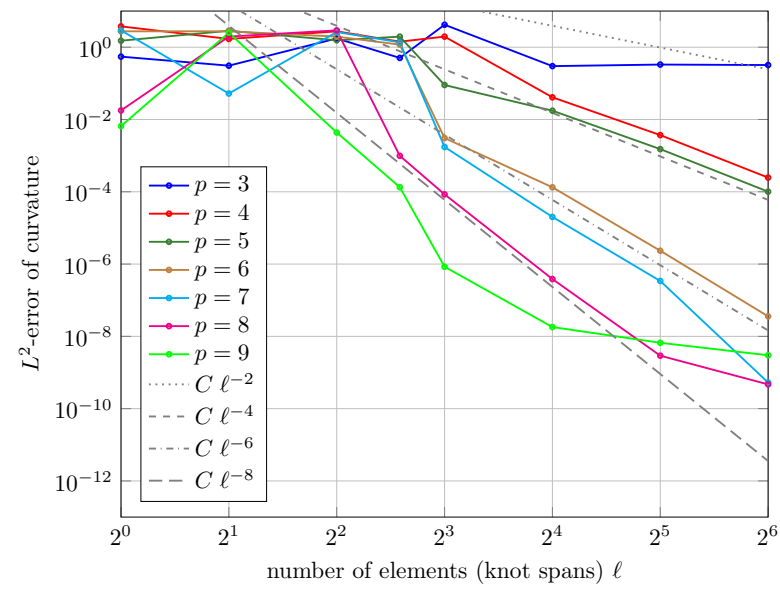

(c) $t=0.01$ : primal formulation $\left(\mathbf{r}_{h}, \mathbf{q}_{h}\right)$

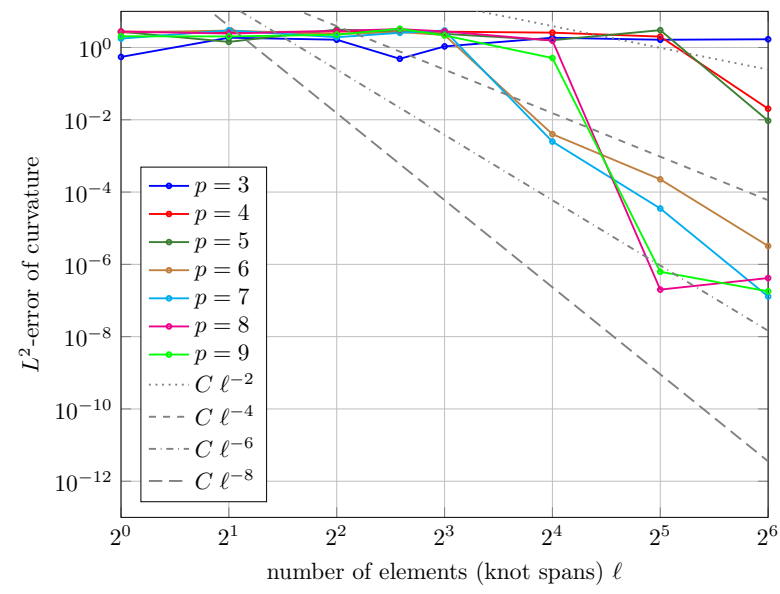

(e) $t=0.001$ : primal formulation $\left(\mathbf{r}_{h}, \mathbf{q}_{h}\right)$

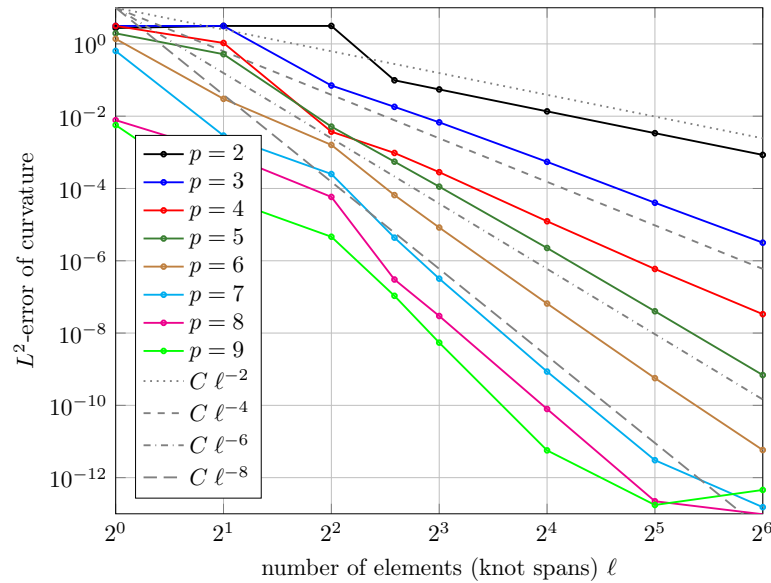

(b) $t=0.1$ : mixed formulation $\left(\mathbf{r}_{h}, \mathbf{q}_{h}, \mathbf{n}_{h}, \mathbf{m}_{h}\right)$

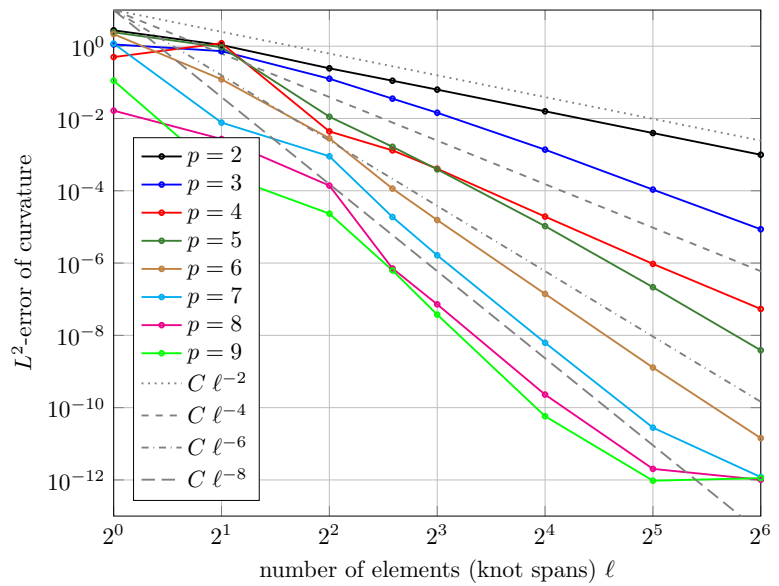

(d) $t=0.01$ : mixed formulation $\left(\mathbf{r}_{h}, \mathbf{q}_{h}, \mathbf{n}_{h}, \mathbf{m}_{h}\right)$

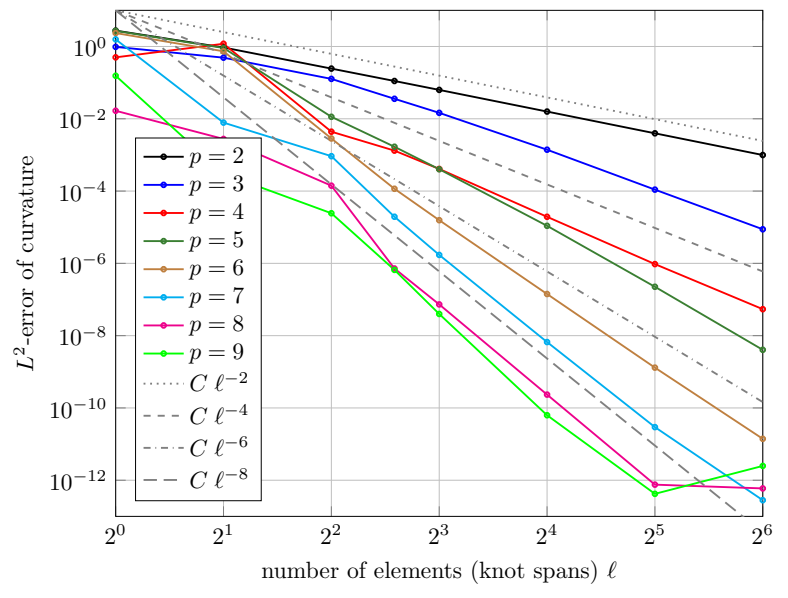

(f) $t=0.001:$ mixed formulation $\left(\mathbf{r}_{h}, \mathbf{q}_{h}, \mathbf{n}_{h}, \mathbf{m}_{h}\right)$

Figure 6: Bending of straight cantilever; investigation of locking for thickness parameter $t=0.1,0.01,0.001$ by comparison of convergence of $L^{2}$-error of curvature $\left\|\boldsymbol{\kappa}^{h}-\boldsymbol{\kappa}^{e}\right\|_{L^{2}}$ for NURBS with degree $p=(2) 3,, \ldots, 9$ and $\ell=1-64$ elements for primal and mixed formulations 


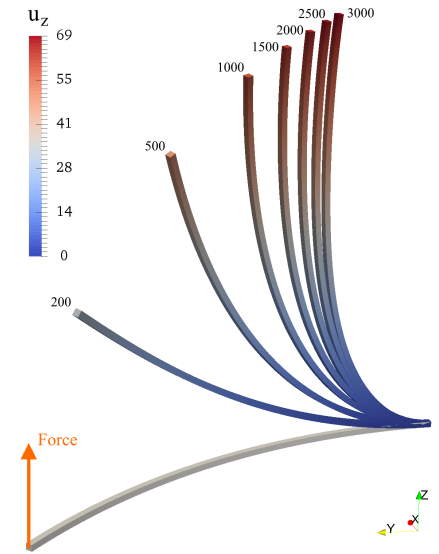

(a) deformed cantilever

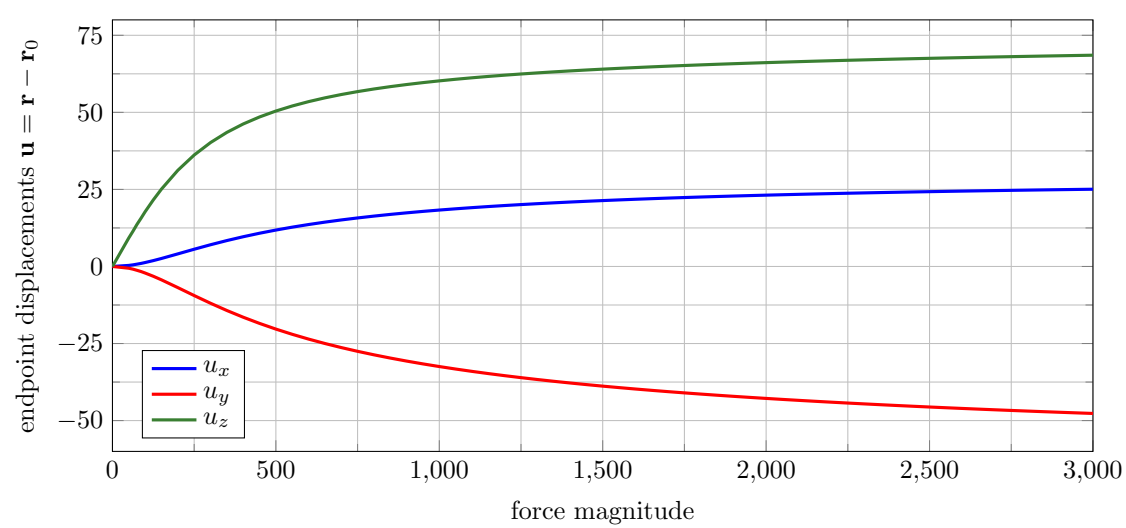

(b) end point displacements

Figure 7: $45^{\circ}$ bend cantilever; deformation of a $45^{\circ}$ bend cantilever rod subject to an increasing end point force, discretized with $p=8, \ell=16$

displacement/rotation-based (primal) formulation is expected due to increasing numerical imbalance of the orders of magnitude of the material parameters $k_{1} G A$ and $E I_{1}$.

To study the convergence behavior of both formulations, primal and mixed, we compute the numerical deformation for the bending into a semi-circle $(\phi=\pi)$, and evaluate the errors compared to the exact solutions for the displaced centerline $\mathbf{r}$ and the curvature $\boldsymbol{\kappa}$ in the $L^{2}$-norm. The results are given in Fig. 5 and Fig. 6, and show the error curves for basis function degree $p=(2) 3,, \ldots, 8$ for $h / k$-refinement from 1 to 64 elements (knot spans). In each row of plots we compare the primal (left) and mixed formulation (right) for $t=0.1,0.01,0.001$ (top, middle, and bottom row).

The results show that for $t=0.1$ the convergence behavior of the primal method matches the expected rates of convergence, which are of order $2\lfloor p / 2\rfloor$ for degree $p\left[27,33\right.$. This holds for both, the $L^{2}$-errrors of displacement and curvature. However, for decreasing the thickness from $t=0.1$ to $t=0.01$ and $t=0.001$ locking becomes more severe. While the convergence rates can still be reached in the limit of very fine discretizations, the error constants increase with a factor $1 / t^{2}$. Also the convergence of the Newton's method used for the solution of the nonlinear system is affected. For smaller $t$ typically more iterations are need, the method diverges more easily, and the error bounds cannot be met. This is reflected by error values larger than 1 in the convergence plots, which typically indicate that the solution did not converge.

In contrast, the accuracy and convergence of the mixed method are not affected by the parameter $t$ and the locking problem is resolved. The error constants are by a factor of $t^{2}$ smaller than for the primal method, and the rate of convergence is even improved to order $2\lceil p / 2\rceil$ for degree $p$.

\section{2. $45^{\circ}$ bend cantilever}

Next, we apply our methods to a reference example for an initially curved rod subject to large displacement, which was studied before in [40, 41. A $45^{\circ}$ bend cantilever beam, i.e. 1/8 section of a full circular arc, is subject to an out-of-plane force of fixed direction and increasing magnitude, which causes combined extension, bending and shearing. The radius of the $\operatorname{arc}$ is 100 units, i.e. the length is $L=25 \pi$, it has a unit rectangular cross-section, and material constants $E=1 \cdot 10^{7}$ and $G=5 \cdot 10^{6}(\nu=0)$. Note that the initial centerline curve can be exactly parameterized by quadratic NURBS here.

Figure 7 shows the deformed cantilever arc for increasing force magnitude from 0 to 3,000. The isogeometric collocation method was applied with NURBS of degree $p=8$ with $\ell=16$ elements (knot spans) and the results correspond very well with the ones presented in [41, see Fig. 7b] For generating a smooth appearance of the load-displacement curves in Fig. $7 \mathrm{~b}$ we have used 60 load steps with constant increments of 50 units. 
Furthermore, we also use this example of an initially curved rod to study the convergence properties of both isogeometric collocation methods for $p$ - and $h / k$-refinement, as well as shear and membrane locking. In Fig. 8 the relative $L^{2}$-errors of the displacement of the centerline $\left\|\mathbf{r}^{h}-\mathbf{r}^{e}\right\|_{L^{2}} / L$ and the $L^{2}$-error of curvatures $\left\|\boldsymbol{\kappa}^{h}-\boldsymbol{\kappa}^{e}\right\|_{L^{2}}$ are given for a load factor of 600 and NURBS degree $p=(2) 3,, \ldots, 9$ with $\ell=1-64$ elements for the primal and the mixed method. As "exact" reference solutions $\mathbf{r}^{e}$ and $\boldsymbol{\kappa}^{e}$ we have used the numerical results for $p=10, \ell=96$. Again, we can observe the theoretical convergence rates of isogeometric collocation methods, which were established in [27, 33. For the displacement/rotation-based method we have convergence rates of order $2\lfloor p / 2\rfloor$ for degree $p$ for both the displacements in Fig. 8a and the curvatures in Fig. 8b. As before, we have convergence rates of order $2\lceil p / 2\rceil$ for degree $p$ for the mixed method for both the displacements in Fig. $8 \mathrm{c}$ and the curvatures in Fig. $8 \mathrm{~d}$. Since the thickness to length ratio is $t / L=1 /(25 \pi) \approx 0.013$ here, shear and membrane locking can be observed for the primal formulation, but they are not as evident as the shear locking in the preceding numerical example, Sect. 7.1. The convergence behavior of the mixed method shows that it also resolves the membrane locking problem.

\subsection{Roll-up of straight cantilever}

In our next example we investigate the roll-up of an initially straight, very slender cantilever rod into a helical spring. The rod has an initial length of $L=1 \mathrm{~m}$ and a circular cross-section with diameter $0.005 \mathrm{~m}$. As material parameterswe use $E=1 \cdot 10^{9} \mathrm{~Pa}, \nu=0.5$, and $k_{1}=k_{2}=5 / 6$. It is clamped at one end and at the other end a moment $\bar{m}_{1}=6 \pi E I_{1}$ and a small, perpendicular force $\bar{n}_{2}=0.36 \mathrm{~N}$ are applied in 24 load steps.

Due to the slenderness of the $\operatorname{rod}(t / L=0.005)$ we have used the mixed method with $p=8, \ell=32, n=40$ for the analysis. As can be seen in Fig. 9a, the applied loads lead to the roll-up of the cantilever into a helical spring with 3 coils. Figure $9 \mathrm{~b}$ shows the highly nonlinear evolution of the displacement of the free end point of the cantilever rod over the 24 load steps. Though no reference solution is available here the results are very reasonable and confirm the robustness of the method for a scenario with very large displacements and rotations.

\subsection{Compression of a helical spring}

The next application deals with the compression of a helical spring. The spring has 8 coils with a radius of $0.1 \mathrm{~m}$ and a height increment of $0.05 \mathrm{~m}$ per winding. The cross-section is circular with a radius of 0.005 $\mathrm{m}$ and material parameters are $E=1 \cdot 10^{9} \mathrm{~Pa}, \nu=0.5, k_{1,2}=5 / 6$. The helix is clamped at the bottom end and a downward-directed force of magnitude $0.5 \mathrm{~N}$ is applied to the top end in 10 load steps, causing a compression and sideward bending of the spring, see Fig. 10a.

The geometry of the spring is circular, and thus it can be parameterized exactly as a single NURBS curve of degree 2. However, since this curve is only $C^{0}$ at internal knots (which would be collocation points), we cannot use this representation directly for the analysis. Alternatively, we have investigated two different approaches: Either, the curve is interpolated by higher order B-Splines with $C^{p-1}$-continuity at internal knots, or the curve is split at $C^{0}$-knots, resulting in a "rod structure" with $32=8 \cdot 4$ curves of degree 2 with knot vectors $\Xi=\{0,0,0,1,1,1\}, n=3$ control points and weights $\{1,1 / \sqrt{2}, 1\}$.

Due to the slenderness of the rods, we employ the mixed formulation for the analysis. For the former approach, we use quartic B-Splines with 128 elements (knot spans) for the interpolation of the helical curve without any further refinements for the analysis $(p=4, \ell=128, n=132)$. For the latter approach, we have $k$-refined the 32 individual curves for the analysis to $p=4, \ell=8, n=12$. The results for both cases are provided in Fig. $10 \mathrm{~b}$ and are visually hardly distinguishable. Comparison to further $p / k$-refinements shows the correctness of this large deformation of the spring, which also validates the approach for coupling of rods, which is $G^{1}$-continuous here. However, using only $p=4, \ell=4, n=8$ in the latter approach gives a different result, as can be seen in Fig. 10b, too. This shows that the nonlinear analysis is sensitive to the usage of a too coarse discretization, but also highlights the advantage of higher continuity isogeometric discretizations. 


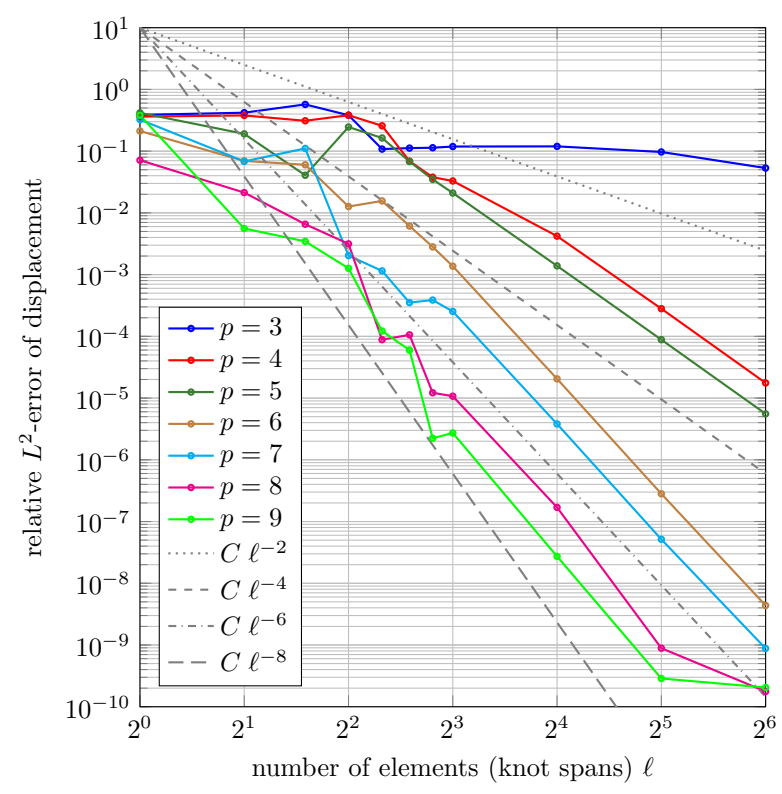

(a) relative $L^{2}$-error of centerline displacement (primal method)

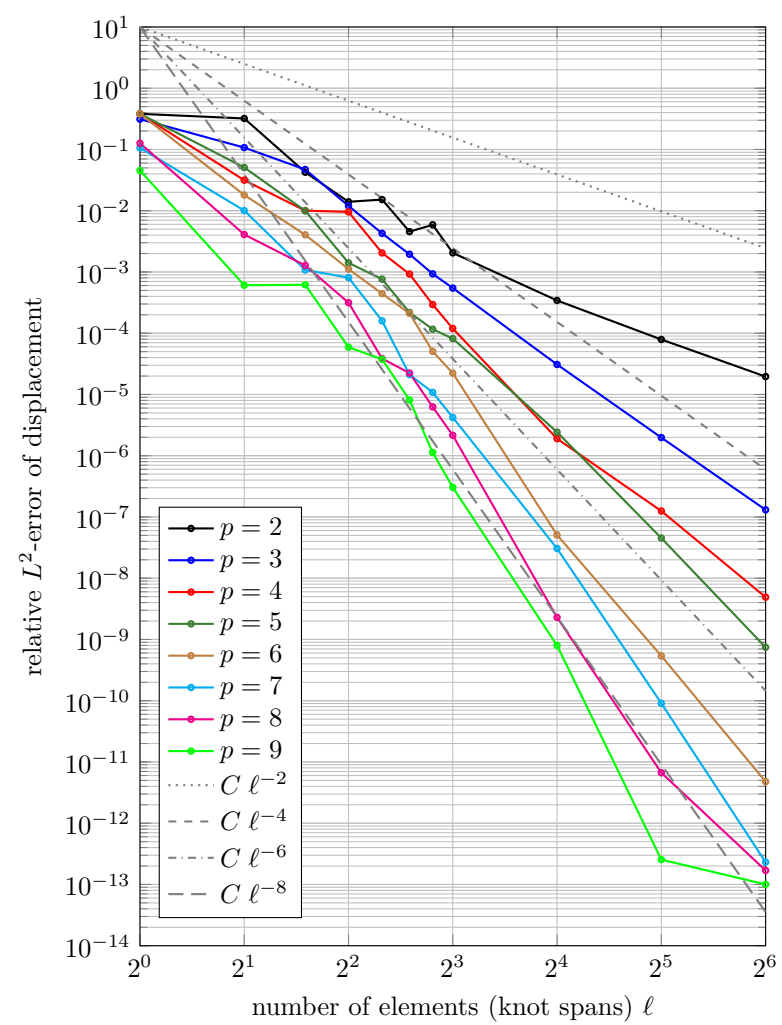

(c) relative $L^{2}$-error of centerline displacement (mixed method)

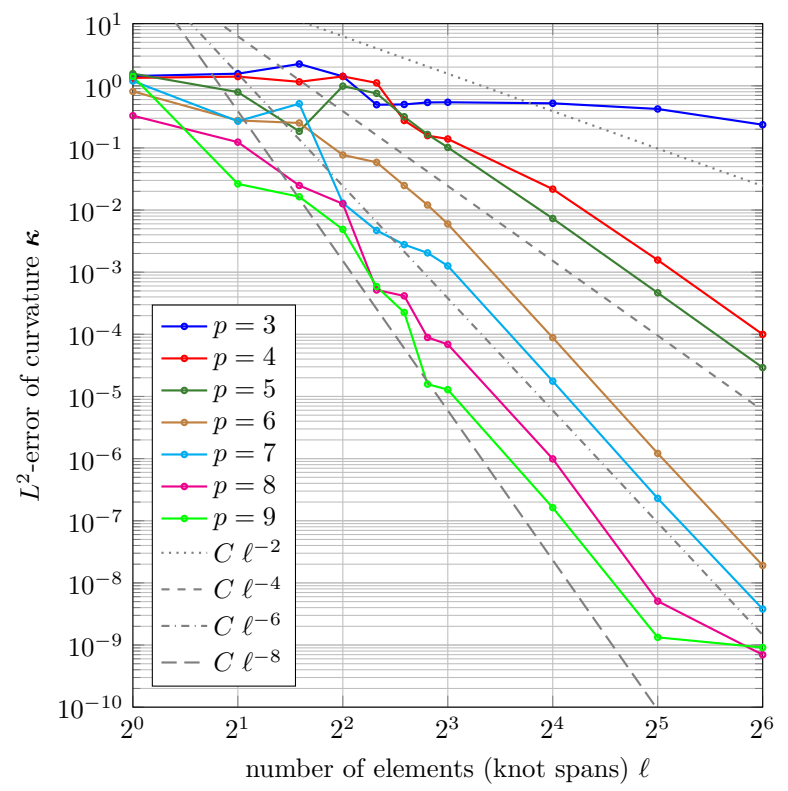

(b) $L^{2}$-error of curvatures (primal method)

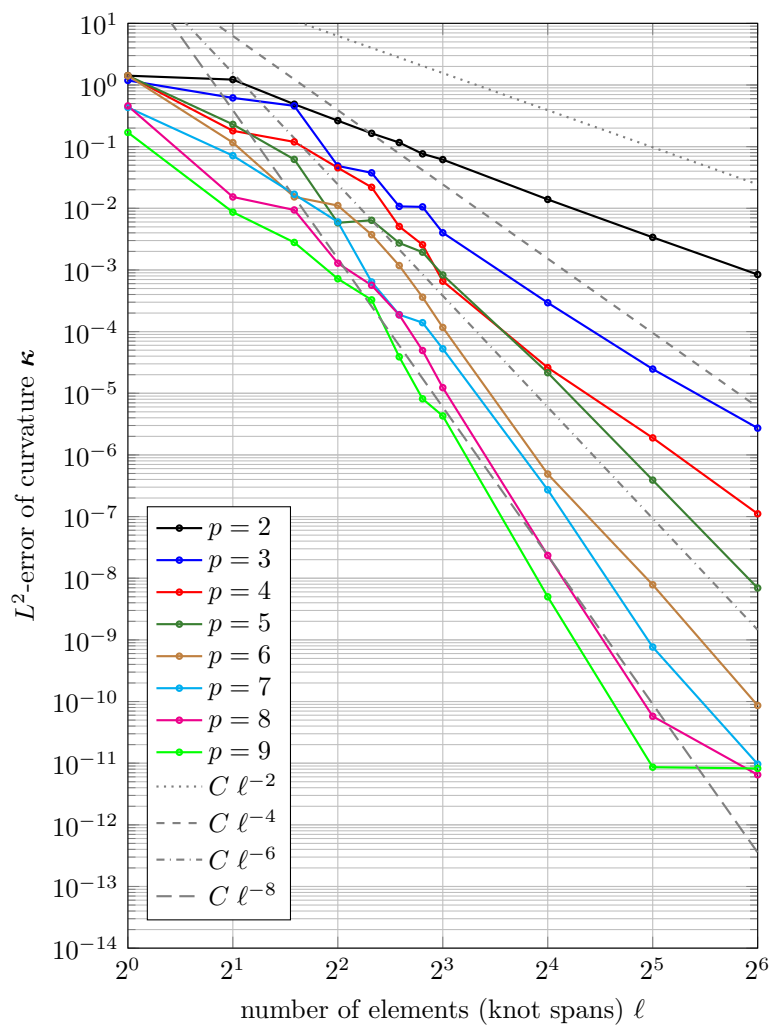

(d) $L^{2}$-error of curvatures (mixed method)

Figure 8: $45^{\circ}$ bend cantilever; convergence of relative $L^{2}$-error of displacement of centerline $\left\|\mathbf{r}^{h}-\mathbf{r}^{e}\right\|_{L^{2}} / L$ and $L^{2}$-error of curvatures $\left\|\boldsymbol{\kappa}^{h}-\boldsymbol{\kappa}^{e}\right\|_{L^{2}}$ for NURBS with degree $p=(2) 3-$,9 and $\ell=1-64$ elements (knot spans) for primal method, (a) and (b) and mixed formulation, (c) and (d) 


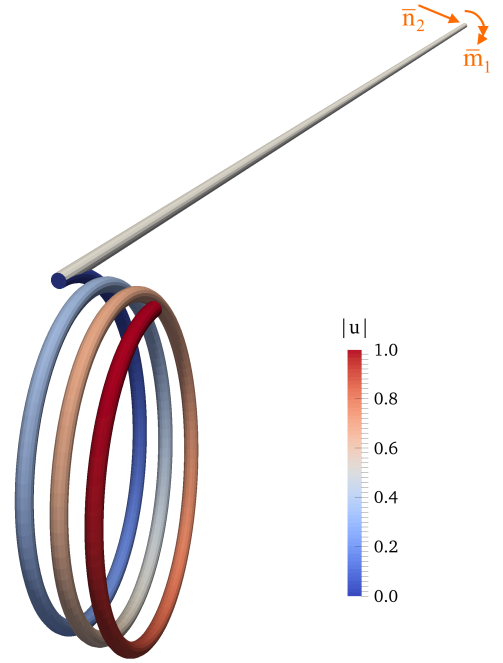

(a) initial configuration and roll-up

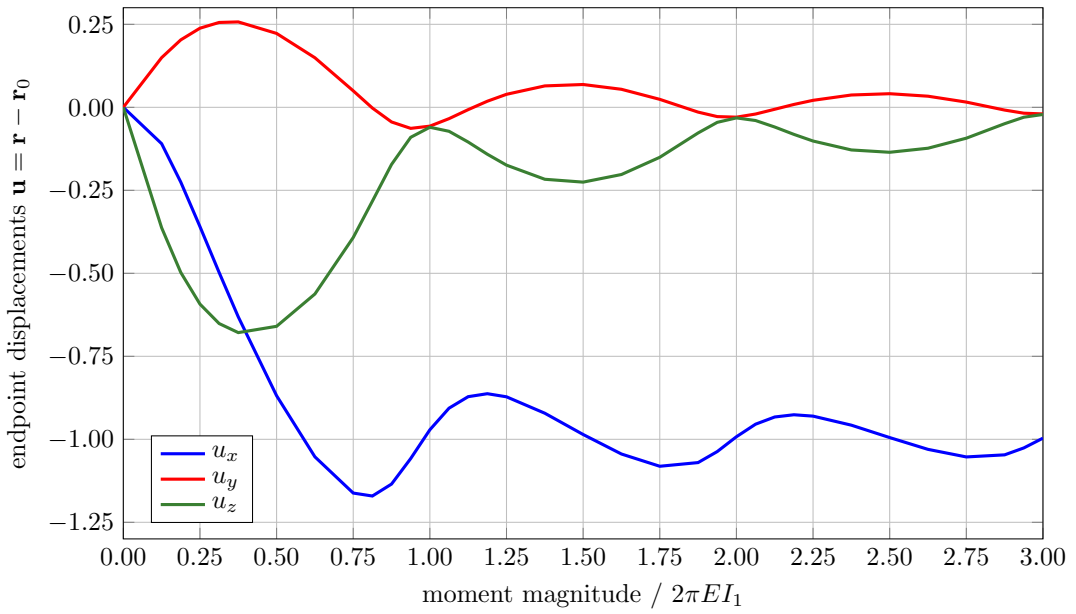

(b) end point displacements

Figure 9: Helical spring; deformation of the helical spring with 8 windings when subject to an end force

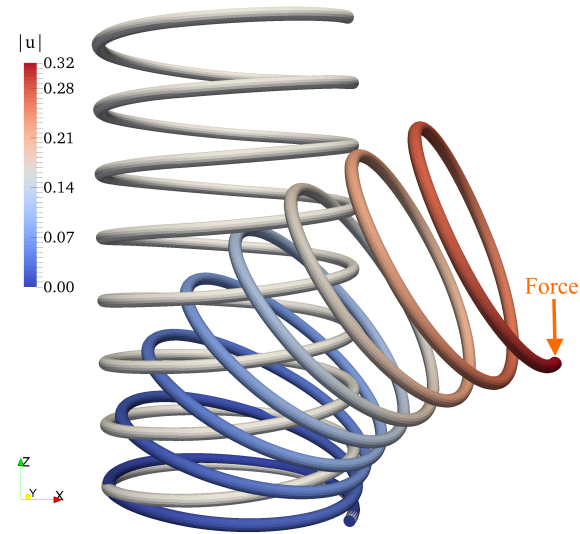

(a) initial configuration and deformed spring

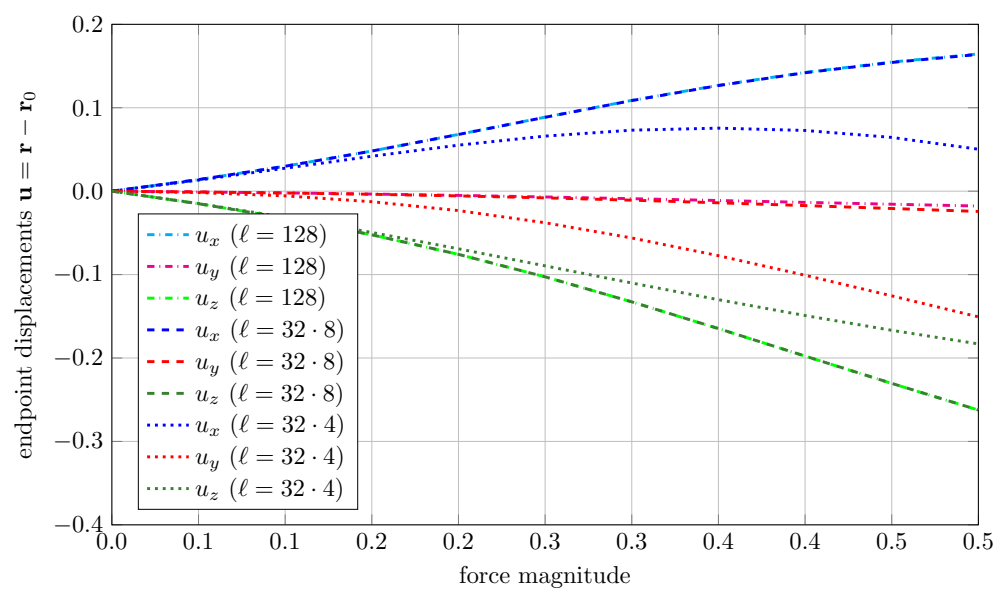

(b) end point displacements for different discretization approaches

Figure 10: Helical spring; deformation of the helical spring with 8 windings when subject to an end force

\subsection{Buckling rod mesh structure}

Finally, we want to introduce an example of a large-scale rod structure. The rod structure, which is shown in Fig. 11a consists of 304 rods with rectangular cross-sections, which are arranged in a 3-dimensional, circular mesh structure. When clamping the structure at the bottom side and applying a uniform, downwarddirected displacement to the top, the structure buckles at the layers with curved rods.

For the analysis we use the primal formulation with $p=8, \ell=8, n=16$ for each rod, resulting in a total of 4,864 control points. In order to capture the highly nonlinear buckling effect, we compress the structure by $1 / 3$ of its height, or two unit cell layers, in around 20 steps with displacement control and adaptively controlled step size. As can be seen in Fig. 11. the vertical layers with curved rods buckle one after another until the structure is fully buckled. The sudden drops of reaction force magnitudes in the compression/force diagram in Fig. 12 indicate when the buckling occurs.

Since the thickness-to-length ratios of the individual rod components of the structure are quite large in this example, we have used the primal method for the analysis. For verification purposes, we have also 

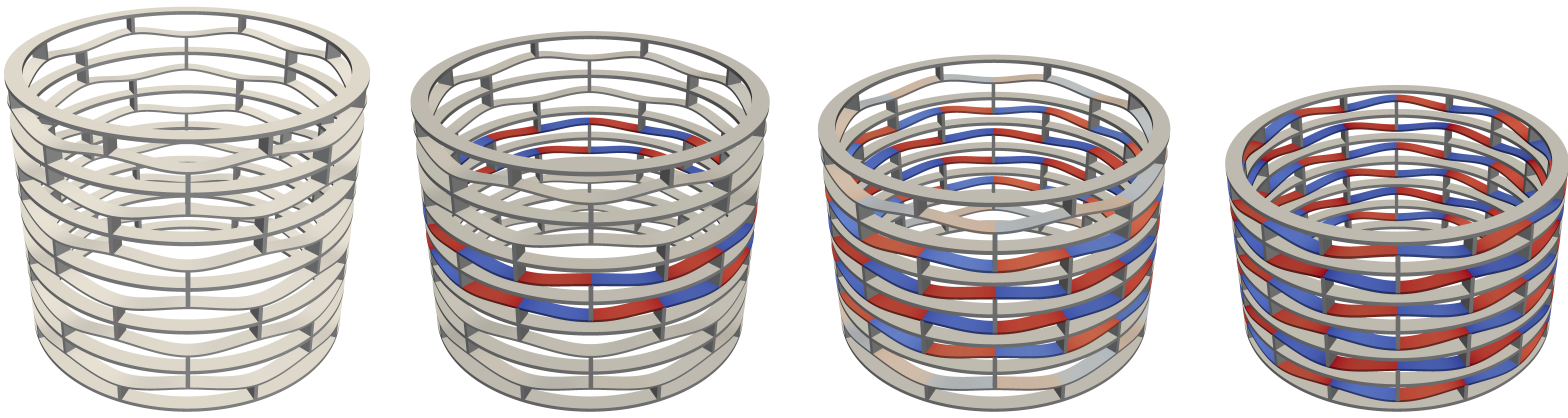

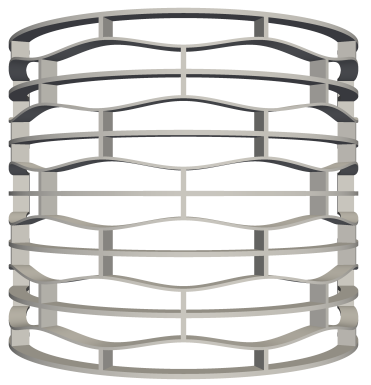

(a) initial configuration

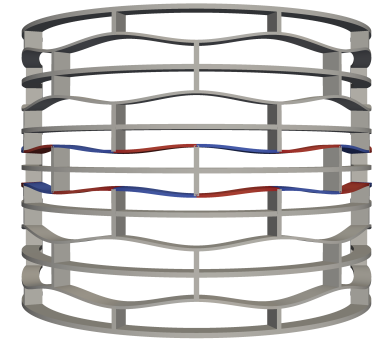

(b) compression 0.6 layers

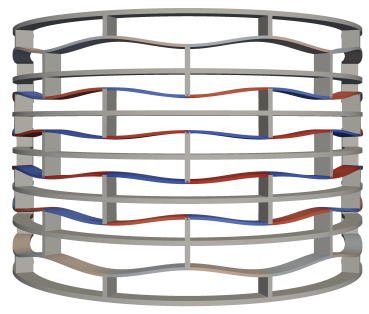

(c) compression 1.2 layers

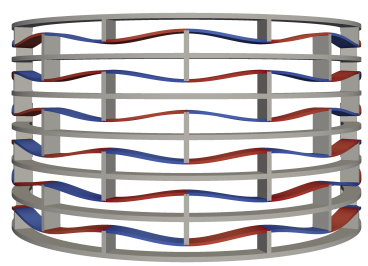

(d) compression 2.0 layers

Figure 11: Buckling mesh; snapshots of buckling mesh structure for increasing compression (coloring by rotational stress $\chi_{3}$ indicates rods which have buckled; for better visibility the back half of the structure is hidden in the bottom views)

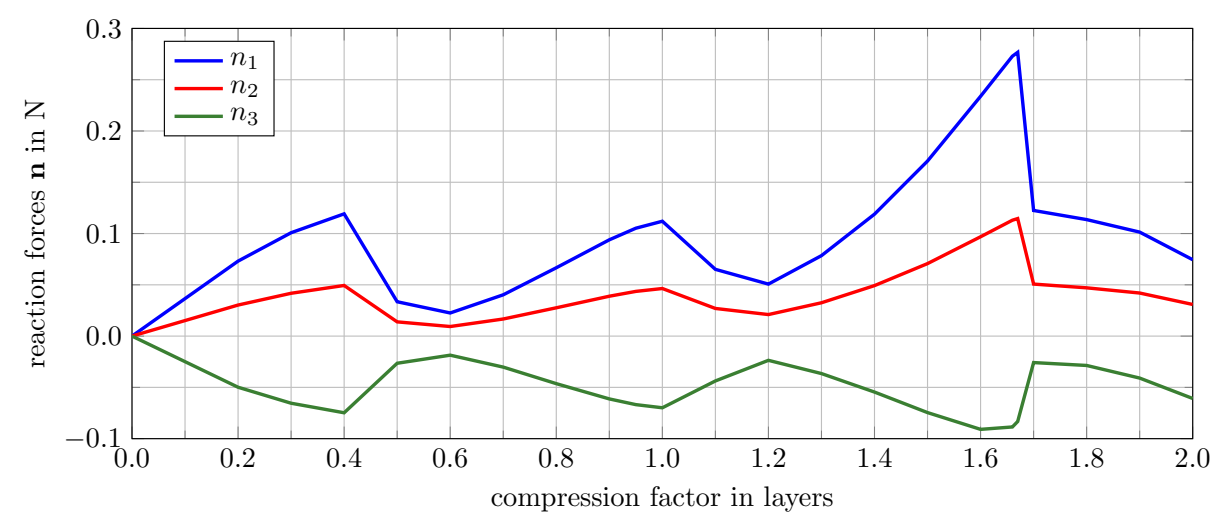

Figure 12: Buckling mesh; reaction forces at bottom side bearings for increasing compression, nonlinear behavior with sudden drops of force indicates buckling

checked the mixed method with $p=8, \ell=8, n=16$ and the reaction forces in Fig. 12 correspond with an accuracy of 5 digits. Overall, this example shows that our rod structure discretization method is also capable of simulating large-scale structures with highly nonlinear deformation behavior, such as buckling.

\section{Summary and conclusions}

In this work we have presented a novel isogeometric collocation method for the Cosserat rod model. First, we have introduced the nonlinear Cosserat rod model, which accounts for finite deformation and rotation of slender elastic rods. The rod's centerline positions and rotation quaternions, which define the crosssection orientations, are parameterized as 3- resp. 4-dimensional NURBS curves. Applying the concept of 
isogeometric collocation to the strong form of the governing equations, i.e. the balance equations for internal forces and moments, we obtain a nonlinear system in terms of the two unknown field variables.

For the simulation of rod structures we have introduced a rigid or quasi- $G^{1}$-coupling approach, which enforces the continuity of centerline positions and changes of rotations, as well as balance of forces and moments at the interfaces. Furthermore, we have also enhanced the isogeometric collocation approach by a mixed method, which is based on an independent discretization of internal forces and moments, and resolves the issue of shear locking for very thin rods.

The presented methods have been carefully studied and validated in a number of numerical applications. Comparison to analytical and numerical reference examples showed that the obtained convergence rates match analytical and numerical rates, which were previously obtained for application of collocation methods to linear models such as the Timoshenko beam and rod [32, 33 .

Altogether, the presented methods and computational results propose isogeometric collocation as a promising alternative to existing discretization methods for Cosserat rods. Since the isogeometric approach allows a straight-forward integration into CAD systems, at least for slender structures such as rods and shells, the method can be easily implemented for engineering applications.

\section{Acknowledgements}

The authors acknowledges support from the SUTD Digital Manufacturing and Design (DManD) Centre, supported by the Singapore National Research Foundation.

\section{Bibliography}

[1] T. J. R. Hughes, J. A. Cottrell, and Y. Bazilevs. Isogeometric analysis: CAD, finite elements, NURBS, exact geometry and mesh refinement. Computer Methods in Applied Mechanics and Engineering, 194(39-41):4135-4195, 2005.

[2] J. A. Cottrell, T. J. R. Hughes, and Y. Bazilevs. Isogeometric Analysis: Toward Integration of CAD and FEA. John Wiley \& Sons, Ltd, 2009.

[3] T. Elguedj, Y. Bazilevs, V.M. Calo, and T.J.R. Hughes. B-bar and F-bar projection methods for nearly incompressible linear and non-linear elasticity and plasticity based on higher-order NURBS elements. Computer Methods in Applied Mechanics and Engineering, 197:2732-2762, 2008.

[4] F. Auricchio, L. Beirão da Veiga, C. Lovadina, and A. Reali. The importance of the exact satisfaction of the incompressibility constraint in nonlinear elasticity: mixed FEMs versus NURBS-based approximations. Computer Methods in Applied Mechanics and Engineering, 199:314-323, 2010.

[5] K. M. Mathisen, K. M. Okstad, T. Kvamsdal, and S. B. Raknes. Isogeometric analysis of finite deformation nearly incompressible solids. Rakenteiden Mekaniikka (Journal of Structural Mechanics), 44(3):260-278, 2011.

[6] N. Cavallini, O. Weeger, M. S. Pauletti, M. Martinelli, and P. Antolín. Effective integration of sophisticated operators in isogeometric analysis with igatools. In B. Jüttler and B. Simeon, editors, Isogeometric Analysis and Applications 2014, volume 107 of Lecture Notes in Computational Science and Engineering, pages 209-230. Springer International Publishing, 2015 .

[7] L. Lorenzis, P. Wriggers, and G. Zavarise. A mortar formulation for 3D large deformation contact using NURBS-based isogeometric analysis and the augmented Lagrangian method. Computational Mechanics, 49(1):1-20, 2011.

[8] E. Brivadis, A. Buffa, B. Wohlmuth, and L. Wunderlich. Isogeometric mortar methods. Computer Methods in Applied Mechanics and Engineering, 284:292 - 319, 2015. Isogeometric Analysis Special Issue.

[9] O. Weeger, U. Wever, and B. Simeon. Nonlinear frequency response analysis of structural vibrations. Computational Mechanics, 54(6):1477-1495, 2014.

[10] W. A. Wall, M. A. Frenzel, and C.. Cyron. Isogeometric structural shape optimization. Computer Methods in Applied Mechanics and Engineering, 197(33 - 40):2976 - 2988, 2008.

[11] A.-V. Vuong D. Fußeder, B. Simeon. Fundamental aspects of shape optimization in the context of isogeometric analysis. Computer Methods in Applied Mechanics and Engineering, 286:313-331, 2015.

[12] Y. Bazilevs, V. M. Calo, T. J. R. Hughes, and Y. Zhang. Isogeometric fluid-structure interaction: theory, algorithms, and computations. Computational Mechanics, 43(1):3-37, 2008.

[13] Y. Bazilevs, L. Beirão da Veiga, J.A. Cottrell, T. J. R. Hughes, and G. Sangalli. Isogeometric analysis: Approximation, stability and error estimates for h-refined meshes. Mathematical Methods and Models in Applied Sciences, 16:1031-1090, 2006.

[14] J. A. Cottrell, T. J. R. Hughes, and A. Reali. Studies of refinement and continuity in isogeometric structural analysis. Computer Methods in Applied Mechanics and Engineering, 196:4160-4183, 2007.

[15] T. J. R. Hughes, J. A. Evans, and A. Reali. Finite element and NURBS approximations of eigenvalue, boundary-value, and initial-value problems. Computer Methods in Applied Mechanics and Engineering, 272:290-320, 2014. 
[16] L. Beirão da Veiga, A. Buffa, G. Sangalli, and R. Vázquez. Mathematical analysis of variational isogeometric methods. Acta Numerica, 23:157-287, 2014.

[17] Y. Bazilevs, V. M. Calo, J. A. Cottrell, J. A. Evans, T. J. R. Hughes, S. Lipton, M. A. Scott, and T. W. Sederberg. Isogeometric analysis using T-splines. Computer Methods in Applied Mechanics and Engineering, 199:229-263, 2010.

[18] M. Pauley, D.-M. Nguyen, D. Mayer, J. Špeh, O. Weeger, and B. Jüttler. The isogeometric segmentation pipeline. In B. Simeon and B. Jüttler, editors, Isogeometric Analysis and Applications 2014, volume 107 of Lecture Notes in Computational Science and Engineering. Springer International Publishing, 2015.

[19] J. A. Cottrell, A. Reali, Y. Bazilevs, and T. J. R. Hughes. Isogeometric analysis of structural vibrations. Computer Methods in Applied Mechanics and Engineering, 195(41-43):5257-5296, 2006.

[20] R. Bouclier, T. Elguedj, and A. Combescure. Locking free isogeometric formulations of curved thick beams. Computer Methods in Applied Mechanics and Engineering, 245-246:144-162, 2012.

[21] S.B. Raknes, X. Deng, Y. Bazilevs, D.J. Benson, K.M. Mathisen, and T. Kvamsdal. Isogeometric rotation-free bendingstabilized cables: Statics, dynamics, bending strips and coupling with shells. Computer Methods in Applied Mechanics and Engineering, 263:127 - 143, 2013.

[22] L. Greco and M. Cuomo. B-spline interpolation of Kirchhoff-Love space rods. Computer Methods in Applied Mechanics and Engineering, 256:251 - 269, 2013.

[23] O. Weeger, U. Wever, and B. Simeon. Isogeometric analysis of nonlinear Euler-Bernoulli beam vibrations. Nonlinear Dynamics, 72(4):813-835, 2013.

[24] J. Kiendl, K.-U. Bletzinger, J. Linhard, and R. Wüchner. Isogeometric shell analysis with Kirchhoff-Love elements. Computer Methods in Applied Mechanics and Engineering, 198(49-52):3902-3914, 2009.

[25] W. Dornisch, S. Klinkel, and B. Simeon. Isogeometric Reissner-Mindlin shell analysis with exactly calculated director vectors. Computer Methods in Applied Mechanics and Engineering, 253:491 - 504, 2013.

[26] N. Nguyen-Thanh, J. Kiendl, H. Nguyen-Xuan, R. Wüchner, K.-U. Bletzinger, Y. Bazilevs, and T. Rabczuk. Rotation free isogeometric thin shell analysis using PHT-splines. Computer Methods in Applied Mechanics and Engineering, 200:34103424, November 2011.

[27] F. Auricchio, L. Beirão da Veiga, T. J. R. Hughes, A. Reali, and G. Sangalli. Isogeometric collocation methods. Mathematical Models and Methods in Applied Sciences, 20(11):2075-2107, 2010.

[28] A. Reali and T.J.R. Hughes. An introduction to isogeometric collocation methods. In G. Beer and S. Bordas, editors, Isogeometric Methods for Numerical Simulation, volume 561 of CISM International Centre for Mechanical Sciences, pages 173-204. Springer, 2015.

[29] D. Schillinger, J. A. Evans, A. Reali, M. A. Scott, and T. J. R. Hughes. Isogeometric collocation: Cost comparison with Galerkin methods and extension to adaptive hierarchical NURBS discretizations. Computer Methods in Applied Mechanics and Engineering, 267:170-232, 2013.

[30] F. Auricchio, L. Beirão da Veiga, T.J.R. Hughes, A. Reali, and G. Sangalli. Isogeometric collocation for elastostatics and explicit dynamics. Computer Methods in Applied Mechanics and Engineering, 249-252:2 - 14, 2012. Higher Order Finite Element and Isogeometric Methods.

[31] R. Kruse, N. Nguyen-Thanh, L. De Lorenzis, and T.J.R. Hughes. Isogeometric collocation for large deformation elasticity and frictional contact problems. Computer Methods in Applied Mechanics and Engineering, 296:73 - 112, 2015.

[32] L. Beirão da Veiga, C. Lovadina, and A. Reali. Avoiding shear locking for the Timoshenko beam problem via isogeometric collocation methods. Computer Methods in Applied Mechanics and Engineering, 241 - 244:38 - 51, 2012.

[33] F. Auricchio, L. Beirão da Veiga, J. Kiendl, C. Lovadina, and A. Reali. Locking-free isogeometric collocation methods for spatial Timoshenko rods. Computer Methods in Applied Mechanics and Engineering, 263:113 - 126, 2013.

[34] J. Kiendl, F. Auricchio, T.J.R. Hughes, and A. Reali. Single-variable formulations and isogeometric discretizations for shear deformable beams. Computer Methods in Applied Mechanics and Engineering, 284:988 - 1004, 2015. Isogeometric Analysis Special Issue.

[35] A. Reali and H. Gomez. An isogeometric collocation approach for Bernoulli-Euler beams and Kirchhoff plates. Computer Methods in Applied Mechanics and Engineering, 284:623 - 636, 2015. Isogeometric Analysis Special Issue.

[36] J. Kiendl, F. Auricchio, L. Beirão da Veiga, C. Lovadina, and A. Reali. Isogeometric collocation methods for the ReissnerMindlin plate problem. Computer Methods in Applied Mechanics and Engineering, 284:489 - 507, 2015. Isogeometric Analysis Special Issue.

[37] S.S. Antman. Nonlinear Problems of Elasticity, volume 107 of Applied Mathematical Sciences. Springer New York, 2005.

[38] S. Eugster. Geometric Continuum Mechanics and Induced Beam Theories, volume 75 of Lecture Notes in Applied and Computational Mechanics. Springer International Publishing, 2015.

[39] J.C. Simo. A finite strain beam formulation. the three-dimensional dynamic problem. Part I. Computer Methods in Applied Mechanics and Engineering, 49(1):55 - 70, 1985.

[40] K.-J. Bathe and S. Bolourchi. Large displacement analysis of three-dimensional beam structures. International Journal for Numerical Methods in Engineering, 14(7):961-986, 1979.

[41] J.C. Simo and L. Vu-Quoc. A three-dimensional finite-strain rod model. Part II: Computational aspects. Computer Methods in Applied Mechanics and Engineering, 58(1):79 - 116, 1986.

[42] K. Arunakirinathar and B. D. Reddy. Mixed finite element methods for elastic rods of arbitrary geometry. Numerische Mathematik, 64(1):13 - 43, 1993.

[43] P. Jung, S. Leyendecker, J. Linn, and M. Ortiz. A discrete mechanics approach to the Cosserat rod theory - part 1: static equilibria. International Journal for Numerical Methods in Engineering, 85(1):31-60, 2011.

[44] A. Lazarus, J.T. Miller, and P.M. Reis. Continuation of equilibria and stability of slender elastic rods using an asymptotic numerical method. Journal of the Mechanics and Physics of Solids, 61(8):1712 - 1736, 2013. 
[45] J. Spillmann and M. Teschner. CoRdE: Cosserat rod elements for the dynamic simulation of one-dimensional elastic objects. In Dimitris Metaxas and Jovan Popovic, editors, Proceedings of the 2007 ACM SIGGRAPH/Eurographics Symposium on Computer Animation, SCA '07, pages 63-72, Aire-la-Ville, Switzerland, Switzerland, 2007. Eurographics Association.

[46] M. Bergou, M. Wardetzky, S. Robinson, B. Audoly, and E. Grinspun. Discrete elastic rods. ACM Trans. Graph., 27(3):63:1-63:12, August 2008.

[47] N. Umetani, R. Schmidt, and J. Stam. Position-based elastic rods. In Vladlen Koltun and Eftychios Sifakis, editors, Eurographics/ACM SIGGRAPH Symposium on Computer Animation, pages 1 - 10. The Eurographics Association, 2014.

[48] S.S. Antman. Dynamical problems for geometrically exact theories of nonlinearly viscoelastic rods. Journal of Nonlinear Science, 6(1):1-18, 1996.

[49] H. Lang, J. Linn, and M. Arnold. Multi-body dynamics simulation of geometrically exact Cosserat rods. Multibody System Dynamics, 25(3):285-312, 2011.

[50] R.L. Bishop. There is more than one way to frame a curve. The American Mathematical Monthly, 82(3):246-251, 1975.

[51] L. A. Piegl and W. Tiller. The NURBS Book. Monographs in Visual Communication. Springer, 1997.

[52] M.-J. Kim, M.-S. Kim, and S.Y. Shin. A general construction scheme for unit quaternion curves with simple high order derivatives. In Proceedings of the 22nd Annual Conference on Computer Graphics and Interactive Techniques, SIGGRAPH '95, pages 369-376, New York, NY, USA, 1995. ACM.

[53] L. Greco and M. Cuomo. An implicit G1 multi patch B-spline interpolation for Kirchhoff-Love space rod. Computer Methods in Applied Mechanics and Engineering, 269:173 - 197, 2014. 